\title{
Network medicine links SARS-CoV-2/COVID- 19 infection to brain microvascular injury and neuroinflammation in dementia-like cognitive impairment
}

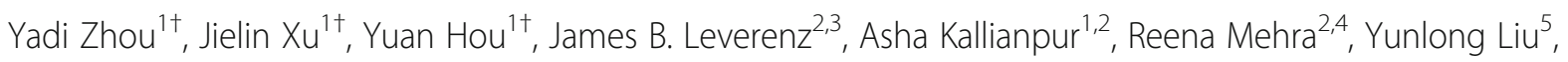
Haiyuan $Y^{6,7,8}$, Andrew A. Pieper ${ }^{9,10,11,12,13,14}$, Lara Jehi ${ }^{2,3}$ and Feixiong Cheng ${ }^{1,2,15^{*}}$ (i)

\begin{abstract}
Background: Dementia-like cognitive impairment is an increasingly reported complication of SARS-CoV-2 infection. However, the underlying mechanisms responsible for this complication remain unclear. A better understanding of causative processes by which COVID-19 may lead to cognitive impairment is essential for developing preventive and therapeutic interventions.

Methods: In this study, we conducted a network-based, multimodal omics comparison of COVID-19 and neurologic complications. We constructed the SARS-CoV-2 virus-host interactome from protein-protein interaction assay and CRISPRCas9-based genetic assay results and compared network-based relationships therein with those of known neurological manifestations using network proximity measures. We also investigated the transcriptomic profiles (including single-cell/ nuclei RNA-sequencing) of Alzheimer's disease (AD) marker genes from patients infected with COVID-19, as well as the prevalence of SARS-CoV-2 entry factors in the brains of AD patients not infected with SARS-CoV-2.

Results: We found significant network-based relationships between COVID-19 and neuroinflammation and brain microvascular injury pathways and processes which are implicated in AD. We also detected aberrant expression of AD biomarkers in the cerebrospinal fluid and blood of patients with COVID-19. While transcriptomic analyses showed relatively low expression of SARS-CoV-2 entry factors in human brain, neuroinflammatory changes were pronounced. In addition, single-nucleus transcriptomic analyses showed that expression of SARS-CoV-2 host factors (BSG and FURIM) and antiviral defense genes (LY6E, IFITM2, IFITM3, and IFNAR1) was elevated in brain endothelial cells of AD patients and healthy controls relative to neurons and other cell types, suggesting a possible role for brain microvascular injury in COVID-19-mediated cognitive impairment. Overall, individuals with the AD risk allele APOE E4/E4 displayed reduced expression of antiviral defense genes compared to APOE E3/E3 individuals.
\end{abstract}

\footnotetext{
* Correspondence: chengf@ccf.org

${ }^{\dagger}$ Yadi Zhou, Jielin Xu and Yuan Hou contributed equally to this work.

'Genomic Medicine Institute, Lerner Research Institute, Cleveland Clinic, Cleveland, OH 44195, USA

${ }^{2}$ Department of Molecular Medicine, Cleveland Clinic Lerner College of Medicine, Case Western Reserve University, Cleveland, OH 44195, USA

Full list of author information is available at the end of the article
} 
Conclusion: Our results suggest significant mechanistic overlap between AD and COVID-19, centered on neuroinflammation and microvascular injury. These results help improve our understanding of COVID-19-associated neurological manifestations and provide guidance for future development of preventive or treatment interventions, although causal relationship and mechanistic pathways between COVID-19 and AD need future investigations.

Keywords: Alzheimer's disease, Brain microvasculature, Cognitive impairment, COVID-19, Dementia, Network medicine, Neuroinflammation, SARS-CoV-2, Single-cell/nucleus

\section{Introduction}

Patients with COVID-19 commonly develop neurologic symptoms and/or complications, such as a loss of taste or smell, stroke, delirium, and rarely new onset seizures $[1,2]$. Based on the experience with other coronaviruses, it was predicted early on that COVID-19 patients might also be at risk for cognitive dysfunction. For example, after the severe acute respiratory syndrome (SARS-CoV-1) outbreak in 2002 and the Middle East respiratory syndrome (MERS) outbreak in 2012, both caused by human coronaviruses (HCoVs), $20 \%$ of recovered patients reported ongoing memory impairment [3]. Evidence now supports similar complications after COVID-19, which due to the global pandemic, is poised to potentially lead to a surge in cases of Alzheimer's-like dementia or other forms of neurocognitive impairment in the near future [4-8].

On the one hand, individuals with dementia (vascular dementia, presenile dementia, and Alzheimer's disease, etc.) were shown to have elevated risks for COVID-19 compared to those without dementia [9]. COVID-19 patients with dementia have elevated mortality rate $[10,11]$, and the most frequent symptoms included hypoactive delirium and functional status worsening [11]. On the other hand, COVID-19 may lead to cognitive impairments, such as shown by poor neuropsychological assessments $[4,12]$ or shown by behaviors or symptoms such as agitation, confusion, inattention, and disorientation [13]. COVID-19 patients admitted to intensive care unit (ICU) have elevated frequency of delirium [14]. In a recent study of a large cohort of more than 236,000 COVID-19 survivors, it was shown that the survivors who required hospitalization, ICU admission, or had encephalopathy during COVID-19 had elevated risks of neurological and psychiatric disorders [8]. Another study using 73,000 non-hospitalized COVID-19 survivors shows various incident sequalae, such as mental health disorders and neurocognitive disorders [15]. Jaywant et al. reported that of 57 recovering COVID-19 patients referred for neuropsychological evaluation before hospital discharge, $81 \%$ had cognitive impairment, including mild, moderate, and severe cognitive impairment [16].

Clarification of the underlying molecular mechanisms of COVID-19-induced cognitive impairment is mandatory for developing effective therapeutic strategies for patients $[9,17,18]$. While some studies have shown that SARS-
CoV-2 may directly infect the brain [19-21], potentially through the olfactory bulb [19], others have shown that SARS-CoV-2 is absent from the brain [22] and cerebrospinal fluid (CSF) [13]. COVID-19 has also been suggested to cause inflammation within the central nervous system (CNS) [18, 22, 23], as well as microvascular injury [22]. For example, the SARS-CoV-2 spike protein, which readily crosses the blood-brain barrier (BBB) [24, 25], induces an inflammatory response within microvascular endothelial cells, leading to BBB dysfunction [25].

Multi-omics datasets for patients with COVID-19, such as bulk and single-cell/nucleus transcriptomic [26], proteomic [27], and interactomic (protein-protein interactions [PPIs]) datasets [28-32], have been generated in order to conduct unbiased investigation of the pathophysiological pathways. We reasoned that network-based drug-disease and disease-disease proximity approaches [33-36], which shed light on the relationship between drugs (and drug targets) and diseases (gene and protein determinants of disease mechanisms in the human PPI network), would provide mechanistic insights into the pathobiology of cognitive dysfunction after SARS-CoV-2 infection, potentially suggesting novel targets for further therapeutic investigation. Thus, we investigated Alzheimer's disease (AD)-like pathobiology associated with SARS-CoV2 infection by using a network-based multimodal omics analytic methodology (Fig. 1). Specifically, we leveraged bulk and single-cell/nuclei RNA-sequencing, proteomics, and interactomics (SARS-CoV-2 virus-host PPIs from mass spectrometry assays and genetic interactions from CRISPRCas9 assays) from COVID-19 and AD patients. We hypothesized that SARS-CoV-2 host factors would be localized in a subnetwork within the comprehensive PPI network and that proteins associated with certain neurologic function would be targeted by the virus either directly, or indirectly through PPIs with virus host factors. As detailed below, our comprehensive analyses show scant evidence of direct brain and neuron damage by COVID-19, but robust evidence for involvement of pathways of neuroinflammation and brain microvascular injury in COVID-19.

\section{Materials and methods SARS-CoV-2 host factor profiles}

In total, we have gathered ten datasets of SARS-CoV-2 (and other HCoVs) target host genes/proteins from 

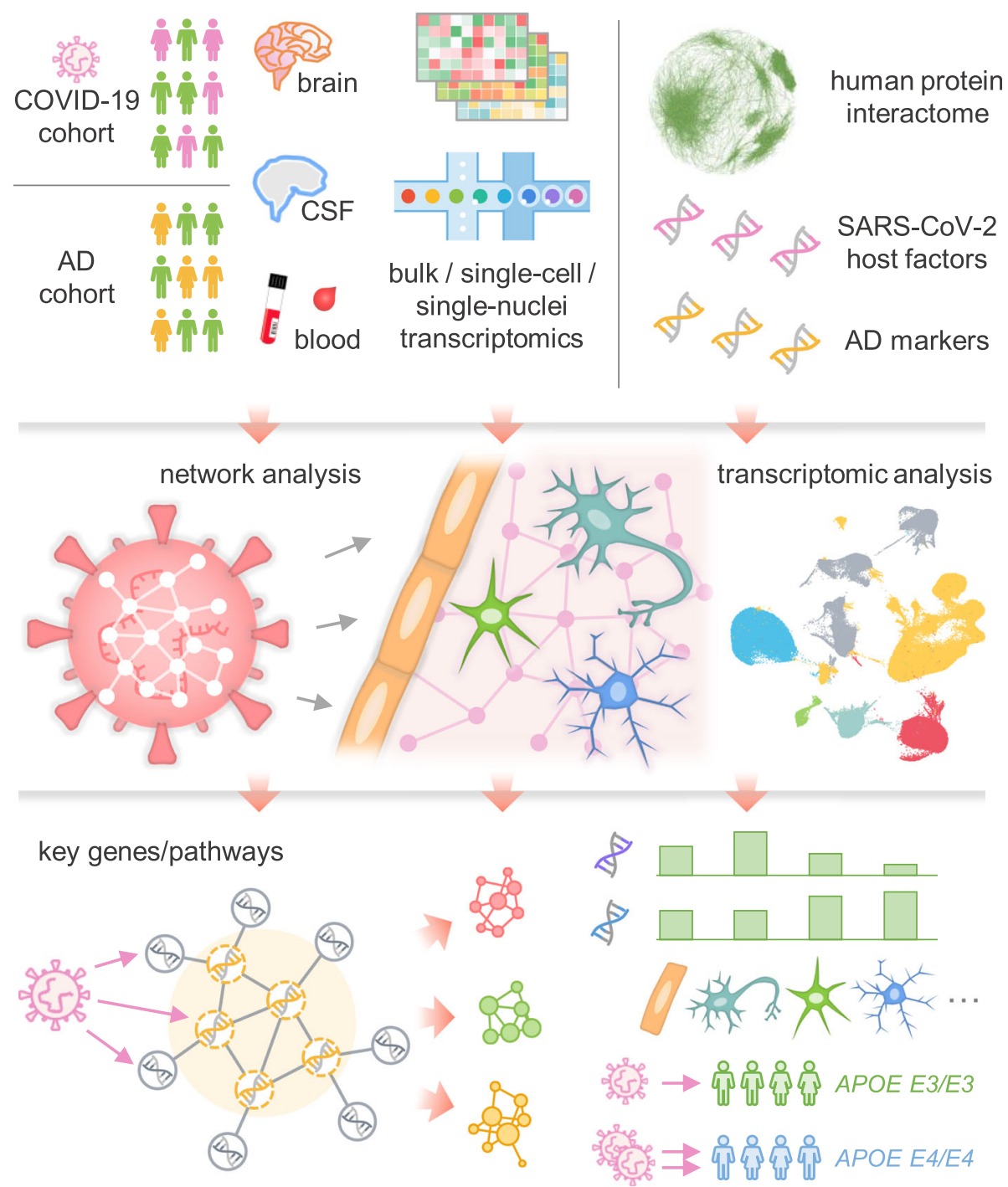

Fig. 1 A diagram illustrating a network-based, multimodal omics analytic framework. We examined the transcriptomes (both bulk and single-cell or single-nucleus) of patients with COVID-19 (blood and cerebrospinal fluid [CSF] samples) or Alzheimer's disease (AD) (brain samples). We also compiled ten SARS-CoV-2 host (human) factor datasets based on CRISPR-Cas9 assays or protein-protein interaction assays, AD blood and CSF markers, and neurological disease-associated genes/proteins. Using network proximity analysis in the human protein-protein interactome, we investigated network-based associations between SARS-CoV-2 host factors and several selected neurological diseases. To understand the potential mechanisms through which SARS-CoV-2 affect the brain, including direct brain invasion, neuroinflammation, and microvascular injury, we examined (1) the expression changes of AD markers in COVID-19 patients, (2) the expression of SARS-CoV-2 host factors in AD patients and healthy individuals at tissues, brain regions, and single-cell/nucleus levels. These transcriptomic analyses were accompanied by network analysis to uncover the potential mechanisms (key genes or pathways) involved in protein-protein interactions. We also compared the susceptibility of SARS-CoV-2 infection among AD patients with different APOE genotypes using the single-nucleus transcriptomic datasets

various data sources (Table S1). Specifically, six of these datasets were based on CRISPR-Cas9 assay results, including (1-2) CRISPR_A549-H and CRISPR_A549-L, based on high $(-\mathrm{H})$ and low $(-\mathrm{L})$ multiplicity of infection of SARS-CoV-2 in A549 cells [30]; (3-5) CRISPR_HuH7SARS2, CRISPR HuH7-229E, CRISPR HuH7-OC43, based on $\mathrm{HuH} 7$ cells infected by SARS-CoV-2, HCoV-229E, and HCoV-OC43, respectively [31]; and (6) CRISPR_VeroE6, based on SARS-CoV-2-infected VeroE6 cells [32]. For the
CRISPR-Cas9-based datasets, we considered the top-100 host factors using the ranking methods described in the respective original publications [30-32]. We also examined the effect of using top-50, -150 , and -200 genes. In addition to the CRISPR datasets, we collected three mass spectrometry-based virus-host PPI datasets [28, 29] for SARS-CoV-2, SARS-CoV-1, and MERS-CoV, named as SARS2-PPI, SARS1-PPI, and MERS-PPI. The last dataset, HCoV-PPI, was from our recent studies [37, 38] containing 
HCoVs target host proteins supported by literature-based evidence. Functional enrichment analyses, including Kyoto Encyclopedia of Genes and Genomes (KEGG) and Gene Ontology (GO) biological process enrichment analyses, were performed using Enrichr [39] for the CRISPR datasets. A list of main SARS-CoV-2 entry factors and proteins involved in antiviral defense was assembled [18], including ACE2, BSG, NRP1, TMPRSS2, TMPRSS11A, TMPRSS11B, FURIN, CTSB, CTSL, LY6E, IFITM1, IFITM2, IFITM3, IFNAR1, and IFNAR2.

\section{Neurological disease gene profiles}

We extracted neurologic disease-associated genes/proteins from the Human Gene Mutation Database (HGMD) [40] and defined a gene to be disease-associated, if it had at least one disease-associated mutation from HGMD reported in the literature. The details of these neurological disease genes can be found in Table S2, including the reported mutations, disease terms used to identify the neurological diseases [41], and original references. For $\mathrm{AD}$, we assembled four datasets from AlzGPS [42], based on our previous work [43] (Table S2). These datasets contain experimentally validated genes (denoted as "seed" genes) in amyloid pathology (amyloid) or tauopathy (tau), as well as high-confidence $A D$ risk genes identified by genome-wide association study (GWAS) [44].

\section{Alzheimer's disease blood and CSF markers}

We compiled a list of AD blood and CSF protein markers from previous studies [45-47], which included 29 blood markers and 31 CSF markers. The expression alteration of these markers in AD or AD-related pathologies, such as tauopathy, were extracted from these studies. The details of these markers can be found in Table S3.

\section{Transcriptomic data analyses}

Two categories of transcriptomic datasets, including three from $\mathrm{AD}$ patients and three from COVID-19 patients, were used (Table S4). These datasets are described below. All single-cell analyses were performed using Seurat v3.1.5 [48] following the processing steps from the original publication of each dataset. Cell types were identified using markers based on the original publications, unless already annotated in the metadata. Differential expression analysis was performed using the "FindMarkers" function from Seurat for the single-cell/nuclei datasets. For the bulk RNA-sequencing dataset, differential expression analysis was performed using edgeR v3.12 [49]. Differentially expressed genes (DEGs) were determined by false discovery rate $(\mathrm{FDR})<0.05$ and $\mid \log _{2}$ foldchange $\mid>0.5$.

\section{GSE147528}

This single-nuclei RNA-sequencing dataset from the superior frontal gyrus and entorhinal cortex regions of
10 males with varying stages of $\mathrm{AD}$ [50] was used to examine the expression of the four key SARS-CoV-2 entry factors: ACE2, TMPRSS2, FURIN, and NRP1, in neurons.

\section{GSE157827}

This single-nuclei RNA-sequencing dataset from the prefrontal cortex region of $12 \mathrm{AD}$ patients and 9 normal controls [51] was used to test the susceptibility of brain endothelial cells to SARS-CoV-2 infection and damage. Six cell types were included: astrocytes, endothelial cells, excitatory neurons, inhibitory neurons, microglia, and oligodendrocytes. The APOE genotypes of these individuals are also available in this dataset.

\section{GSE138852}

This single-nuclei RNA-sequencing dataset from the entorhinal cortex of individuals with $\mathrm{AD}(n=6)$ and healthy controls $(n=6)$ [52] was used to validate the findings of the expression of SARS-CoV-2 entry factors in brain endothelial cells. Six cell types were included: astrocytes, endothelial cells, neurons, microglia, oligodendrocytes, and oligodendrocyte progenitor cells.

\section{GSE157103}

This bulk RNA-sequencing dataset of 125 peripheral blood mononuclear cell (PBMC) samples [53] was used to examine the expression spectrum of $\mathrm{AD}$ blood biomarkers. DEGs were analyzed by disease severity conditions: 66 intensive care unit (ICU) patients $(n=50$ COVID-19 patients vs. $n=16$ non-COVID-19 patients), 59 non-ICU patients ( $n=49$ COVID-19 patients vs. $n=10$ non-COVID-19 patients), and all 125 patients. Adjustments for the effects of age and sex were made.

\section{GSE149689}

This single-cell RNA-sequencing PBMC dataset of 6 samples from severe COVID-19 patients, 4 samples from mild COVID-19 patients, and 4 samples from healthy controls [54] was used to examine the expression spectrum of $\mathrm{AD}$ blood markers. 13 cell types were included in this dataset: $\operatorname{lgG}{ }^{-} \mathrm{B}$ cells, $\operatorname{lgG}^{+} \mathrm{B}$ cells, $\mathrm{CD} 4^{+} \mathrm{T}$ cell effector memory (EM)-like cells, $\mathrm{CD}^{+} \mathrm{T}$ cell nonEM-like cells, $\mathrm{CD}^{+} \mathrm{T}$ cell EM-like cells, $\mathrm{CD}^{+} \mathrm{T}$ cell non-EM-like cells, dendritic cells, monocytes, intermediate monocytes, nonclassical monocytes, natural killer cells, platelets, and red blood cells.

\section{GSE163005}

This single-cell RNA-sequencing CSF dataset [55] was used to examine the expression spectrum of AD CSF markers. This neuro-COVID-19 dataset contains 8 COVID-19 patients, 9 multiple sclerosis (MS) patients, 9 idiopathic intracranial hypertension (IIH) patients, and 5 viral encephalitis (VE) patients. Based on the original 
publication, the cells were categorized into three major cell groups of $\mathrm{T}$ cells, dendritic cells, and monocytes. Four comparisons were performed for each major cell group: COVID-19 vs. MS, COVID-19 vs. IIH, COVID-19 vs. VE, and COVID-19 vs. non-COVID-19 (MS, IIH, and VE).

\section{Human protein-protein interactome}

The human protein-protein interactome was from our previous studies [33, 34, 56, 57], and contains 17,706 protein nodes and 351,444 unique PPI edges. Each PPI edge has one or more source information of five categories of evidence from publicly available databases and datasets: protein complexes identified by robust affinity purification-mass spectrometry from BioPlex V2.016 [58]; binary PPIs discovered by high-throughput yeast two-hybrid systems in three datasets [33, 59, 60]; signaling networks revealed by low-throughput experiments from SignaLink2.0 [61]; low-throughput or high-throughput experiments uncovered kinase-substrate interactions from KinomeNetworkX [62], Human Protein Resource Database (HPRD) [63], PhosphoNetworks [64], PhosphositePlus [65], DbPTM 3.0 [66], and Phospho.ELM [67]; and PPIs curated from literatures identified by yeast two-hybrid studies, affinity purification-mass spectrometry, low-throughput experiments, or protein three-dimensional structures from BioGRID [68], PINA [69], Instruct [70], MINT [71], IntAct [72], and InnateDB [73]. Inferred PPIs derived from evolutionary analysis, gene expression data, and metabolic associations were excluded.

\section{Network analyses}

We used network proximity metrics to quantify the network associations of two gene/protein modules. The "shortest" proximity measure was used to evaluate the overall average distance among all genes in the neurological disease gene sets and the SARS-CoV-2 host factor profiles:

$$
\left\langle d_{A B}^{S}\right\rangle=\frac{1}{\|A\| \times\|B\|} \sum_{a \in A, b \in B} d(a, b)
$$

where $d(a, b)$ represents the shortest path length between gene $a$ from module $A$ and $b$ from module $B$ in the human protein-protein interactome. "closest" proximity measure was used to quantify the distance among the $\mathrm{AD}$ markers and the DEGs from the COVID-19 omics datasets focusing on the genes that are closest to the genes in the other module:

$$
\left\langle d_{A B}^{C}\right\rangle=\frac{1}{\|\mathrm{~A}\|+\|B\|}\left(\sum_{a \in A} \min _{b \in B} d(a, b)+\sum_{b \in B} \min _{a \in A} d(a, b)\right)
$$

All network proximities were converted to $\mathrm{Z}$ scores based on permutation tests of 1000 repeats:

$$
Z_{d_{A B}}=\frac{d_{A B}-\overline{d_{r}}}{\sigma_{r}}
$$

where $\overline{d_{r}}$ and $\sigma_{r}$ are the mean and standard deviation of the proximities, respectively. In each degreecontrolled permutation test, two protein sets were randomly selected which had similar degree distribution to that of the original two protein sets to reduce the effect of degree biases based on our previous studies [33, 38, 56]. A P value was computed using the permutation test accordingly. $\mathrm{P}$ values were corrected for FDR at 0.05 . Gene set pairs with FDR $<0.05$ and $Z<-1.5$ were considered significantly proximal.

The largest connect component (LCC) was computed by NetworkX [74]. Significance of LCC was computed in the same way as the network proximity using permutation test repeated 1000 times. Eigenvector centrality [75] of the nodes in the networks was computed using Gephi 0.9.2 [76] to evaluate the influence of the nodes considering the importance of their neighbors.

\section{Tissue and brain region expression specificity}

We retrieved the transcriptomic data in raw count and transcripts per million (TPM) from the GTEx v8 release [77] for 33 human tissues and 13 brain regions, and examined expression across different tissues and brain regions. At the tissue level, the brain regions were combined as one "brain" tissue. We first defined a gene to be tissue- or brain region-expressed if it had a count per million $(\mathrm{CPM}) \geq 0.5$ in over $90 \%$ samples. Then, to quantify the significance of the expression of a gene in a tissue or brain region, we normalized its expression using the $\mathrm{z}$ score method.

\section{Innate immune genes}

We retrieved a list of 1031 human innate immunity genes from InnateDB [73], which were associated in the published literature with roles in innate immunity.

\section{Statistical analysis and network visualization}

Python package SciPy v1.3.0 [78] was used for the statistical tests unless specified otherwise. $P<0.05$ (or FDR $<0.05$ when applicable) was considered statistically significant throughout the study. Networks were visualized with Gephi 0.9.2 [76] and Cytoscape 3.8.0 [79].

\section{Results}

A network-based, multimodal omics analytic framework In this study, we present a network-based, multimodal omics (including bulk and single-cell/nuclei RNAsequencing, proteomics, and interactomics) analysis method for investigating the underlying mechanisms of COVID-19-associated cognitive dysfunction or impairment. We hypothesized that for COVID-19 to have neurological 
impacts in the host CNS, its host factors (genes/proteins) should be localized in the corresponding subnetwork within the human PPI network, and either directly target the neurological disease-associated genes/proteins or indirectly affect them through PPIs (Fig. 1). We utilized single-cell/ nuclei RNA-sequencing data from both COVID-19 patients with neurological manifestations (neuro-COVID-19) and brains of AD patients not infected by SARS-CoV-2, brainregion-specific gene expression data from the GTEx database [77], SARS-CoV-2 virus-host PPIs from mass spectrometry assays, genetic interactions from CRISPRCas9 assays (Table S1), and disease-related genetic data (Table S2).

We compiled ten virus-host interaction datasets across SARS-CoV-2, SARS-CoV-1 and MERS-CoV, and other common $\mathrm{HCoVs}$, including six datasets from CRISPRCas9 assays and four datasets for virus-human PPIs (Table S1). Functional enrichment analyses of each dataset revealed that virus-host PPIs and host factors are significantly enriched in pathways well-known to be involved in SARS-CoV-2 infection and related immune responses (Supplementary Results, Fig. S1). Using these datasets, we computed their network associations with ten neurological diseases or conditions. To determine whether brain damage was caused by SARS-CoV-2 direct infection of the brain, we evaluated expression levels of SARS-CoV-2 entry genes at brain region and brain single-cell levels. Neuroinflammation was evaluated by identifying alterations in expression of $\mathrm{AD}$ blood and CSF biomarkers in COVID-19 patients using data from peripheral blood mononuclear cell (PBMC) and CSF samples (neuro-COVID-19 dataset). Lastly, microvascular injury was evaluated by examining the expression of SARS-CoV-2 entry factors and antiviral defense genes in brain endothelial cells of $\mathrm{AD}$ and healthy control samples. We also compared the expression of SARS-CoV-2 entry factors and antiviral defense genes in individuals with different $A P O E$ genotypes.

\section{Strong network-based relationships of COVID-19 to neurological manifestations}

We assembled experimentally validated gene/protein profiles for ten neurological diseases or conditions, including $\mathrm{AD}$, amyotrophic lateral sclerosis, cognitive decline, dementia, frontotemporal dementia, multiple system atrophy, neuronal ceroid lipofuscinosis, Parkinson's disease (PD), spinal muscular atrophy, and spinocerebellar ataxia (Table S2). First, we quantified the network distance of the SARS-CoV-2 host factor datasets and neurological diseases in the human protein-protein interactome. A close network distance between SARS-CoV-2 host factors and neurological disease-associated genes/proteins suggests related or shared mechanistic pathways between COVID-19 and a specific neurological disease [38]. Using state-of-the- art network proximity measures (see the "Materials and methods" section), we evaluated the network-based relationship for the gene/protein sets between virus-host factors and each disease/condition under the human interactome network model (Fig. 2a and Fig. S2). We found significant proximities between the SARS-CoV-2 virus-host interactome (including PPIs and genetic interactions) and genes associated with neurological diseases in the human interactome network (average $Z=-1.82$ ). The SARS-CoV2 virus-host PPIs (average $Z=-2.54$ ) showed more significant network proximities (white circles, Fig. 2a) compared to CRISPR-Cas9-derived host factors (average $\mathrm{Z}=-1.34$ ). The top three neurological diseases or conditions with the smallest network proximities to SARS-CoV-2 were AD (average $\mathrm{Z}=-2.75)[9,17]$, cognitive decline (average $\mathrm{Z}=$ - 2.77), and PD (average $Z=-2.94$ ). Recent case reports of COVID-19 patients developing parkinsonism suggest that COVID-19 patients may have an increased risk of PD later in life [80]. We noticed that amyloid pathology has significant network proximity (average $\mathrm{Z}=-1.55$ ) with the PPI datasets. However, there are no significant network-based relations between tauopathy-related genes and the SARS$\mathrm{CoV}-2$ interactome. One possible explanation is the incompleteness of genes/proteins related to tauopathy in the datasets. In addition to SARS-CoV-2, HCoV-229E also showed a significant network proximity to neurological diseases, suggesting a common association between coronaviruses and cognitive dysfunction [81].

\section{A network-based relationship between COVID-19 and Alzheimer's disease}

To examine further why cognitive impairment has such significant network-based association with the SARSCoV-2 interactome, we focused on AD and visualized the PPIs among AD seed genes/proteins (Fig. 2b, green nodes) and host genes/proteins illustrated by the four SARS-CoV-2 virus-human PPI datasets (Fig. 2b, blue nodes). We found a large number of PPIs among these proteins, including multiple blood and CSF biomarkers and SARS-CoV-2 entry factors (nodes with gene symbols). Here, we discuss several markers that may have important roles in COVID-19-associated AD (Table S5) according to network measures (connectivity and eigenvector centrality $[E C]$ ), including vascular cell adhesion protein 1 (VCAM1) (connectivity $\mathrm{K}=73$ ), ras-related protein Rab-7a (RAB7A) (K = 30), and transforming growth factor beta 1 (TGFB1) $(\mathrm{K}=10)$. These proteins have high EC values, a measure of potential node (gene/ protein) influence on the network that considers the influence of its neighbors: VCAM1 EC $=0.59$ (rank 6 out of $153 \mathrm{AD}$ genes/proteins), RAB7A EC $=0.17$ (rank 25), and TGFB1 $\mathrm{EC}=0.19$ (rank 22).

VCAM1 is located at the endothelial cell surface and is activated by cytokines [82]. It is also an AD biomarker 
a

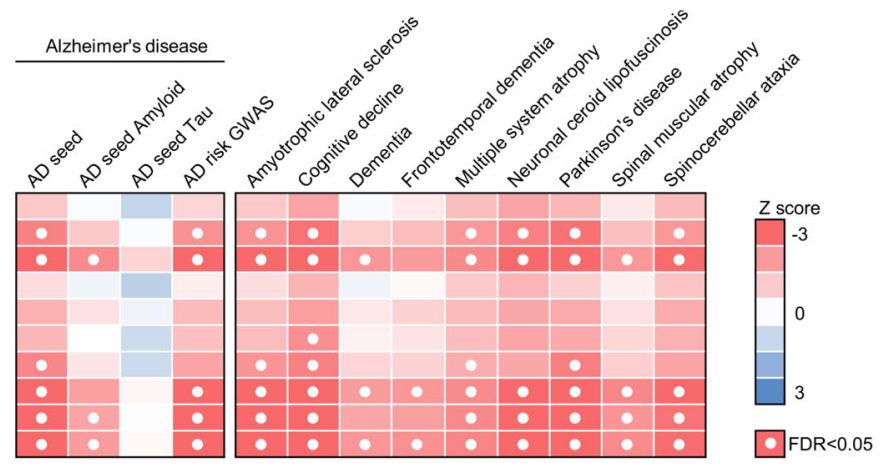

b

CRISPR

CRISPR_HuH7-229E CRISPR_HuH7-OC43 CRISPR_HuH7-SARS2 CRISPR_VeroE6 HCoV-PPI MERS-PPI SARS1-PPI SARS2-PPI

$\therefore \therefore \cdots$
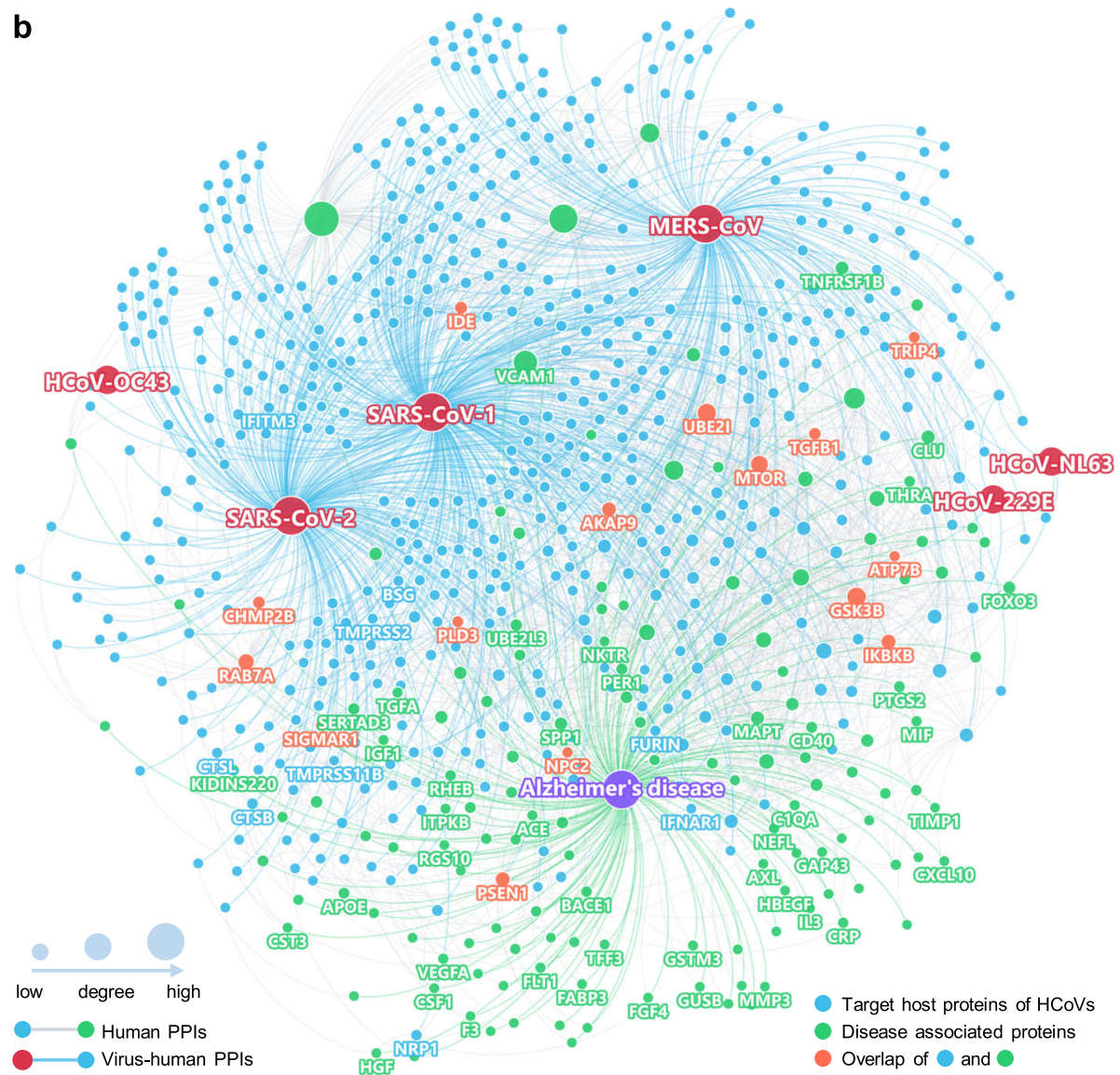

Fig. 2 A network landscape of COVID-19 and neurological diseases. a Network proximity analysis shows strong network associations between COVID-19 and neurological diseases. Heatmap shows the "shortest" network proximities in Z score (see the "Materials and methods" section). Smaller Z scores indicate smaller network proximities between the two gene sets. $\mathbf{b}$ Protein-protein interaction network of the SARS-CoV-2 and other human coronaviruses host factors and the Alzheimer's disease-associated genes/proteins. SARS-CoV-2 entry factors, antiviral defense genes, and $A D$ biomarkers are highlighted by their gene symbols

with elevated expression in the blood [83, 84] and CSF $[45,46]$ of AD patients. VCAM1 levels were also significantly associated with the severity of dementia and structure changes of white matter [84], and brain endothelial VCAM1 at the blood-brain barrier has been proposed as a target for treating age-related neurodegeneration [85]. Serum VCAM1 levels were also significantly elevated in severe COVID-19 patients compared to mild patients and controls, and significantly decreased in the convalescence phase compared to severe patients [86]. Notably, VCAM1 also plays an important role in COVID-19-induced vasculitis [87]. RAB7A is a direct target of non-structural protein 7 (nsp7) of SARS-CoV-2 [29], and also one of the top host factors in CRISPR-Cas9-based SARS-CoV-2 datasets. RAB7A knockout reduces cell surface angiotensinconverting enzyme 2 (ACE2) levels, which thereby reduces 
SARS-CoV-2 entry into cells [30]. RAB7A is also a potential AD biomarker whose blood expression level is positively associated with high memory test performance [47]. TGFB1 is a cytokine that controls cell growth and differentiation [88, 89] and a potential $\mathrm{AD}$ marker with decreased expression in the blood of $\mathrm{AD}$ patients [47]. The anti-inflammatory and neuroprotective role of TGFB1 against $\mathrm{AD}$ has already been demonstrated in animal models [90, 91]. Using bulk RNA-sequencing data from PBMC samples of COVID-19 patients, we also found that TGFB1 expression was significantly decreased in both mild COVID-19 patients and those requiring ICU level care, as compared to non-COVID-19 patients (Table S3).

Altogether, these results encouraged us to explore further the pathological relationships between COVID-19 and $\mathrm{AD}$ and to identify potential pathological pathways by which SARS-CoV-2 infection could lead to AD-like dementia.

\section{Neuroinflammation-mediated association between neuro- COVID-19 and AD}

We next turned to investigate whether neuroinflammation was a shared mechanism between COVID-19 and AD by investigating the expression levels of well-known AD blood and CSF marker genes in COVID-19 patients with neurological manifestations (neuro-COVID-19). To this end, we compiled a list of blood and CSF protein markers for AD from previous studies [45-47] (Table S3) with their expression alterations in $\mathrm{AD}$ or $\mathrm{AD}$-related pathologies. We then examined their expression in COVID-19 patient PBMC [53, 54] and CSF [55] samples. We performed differential expression analyses for the PBMC bulk RNA-sequencing dataset [53] of COVID-19 patients vs. non-COVID-19 patients. For the other singlecell level PBMC dataset [54], we compared mild / severe COVID-19 patients to healthy controls. We used an additional single-cell RNA-sequencing dataset generated from CSF samples of neuro-COVID-19 patients with welldefined neurological manifestations [55].

We first examined the degree of overlap between $\mathrm{AD}$ markers and differentially expressed genes (DEGs) in PBMCs or CSF from COVID-19 patients and found significant overlap in CSF monocytes (FDR $=0.015$, Fisher's exact test, Table S3), but not in PBMCs $($ FDR $=1.00$, Table S3). We further computed the network proximities of the AD markers and DEGs and found that blood markers and DEGs from PBMCs do not show significant network proximities, whereas CSF markers and DEGs from CSF monocytes were significantly proximal (Table S3, $\mathrm{Z}=-3.69$, FDR $=0.009$ ). We also examined the overlaps of the immune genes in the protein markers and the DEGs and found that the CSF markers (immune genes) still have strong overlap and close network proximity to the CSF monocyte DEGs (immune genes) in COVID-19 (Fisher's exact test FDR $=0.035$; network proximity $\mathrm{Z}=-4.39$, FDR $<0.001)$ compared to blood protein markers and blood DEGs (Fisher's exact test FDR $=1.00$, proximity $\mathrm{Z}=-2.16$, proximity $F D R=0.020$ ). Altogether, we found a more significant network-based relationship between COVID-19 and $\mathrm{AD}$ in CSF (including monocytes) compared to PBMCs from COVID-19 patients. We next examined the overall expression spectrum of these markers in both PBMCs and CSF (Fig. 3a, b).

In PBMCs, the expression of several AD markers was altered by SARS-CoV-2 infection, such as TGFB1, SERTA domain-containing protein 3 (SERTAD3), glutathione S-transferase M3 (GSTM3), kinase D-interacting substrate of $220 \mathrm{kDa}$ (KIDINS220), natural killer tumor recognition sequence (NKTR), arylsulfatse $\mathrm{B}(A R S B)$, and insulin-like growth factor 1 (IGF1) (Fig. 3a). Some of the markers have expression changes in the same direction in COVID-19 and AD or AD-related pathologies, including TGFB1, GSTM3, and NKTR. Using the PBMC single-cell RNA-sequencing data, we found that prostaglandin-endoperoxide synthase 2 (PTGS2) and period circadian regulator 1 (PER1) were significantly elevated in monocytes (Fig. S3) of severe COVID-19 patients. PTGS2 expression was also elevated in the bulk PBMC dataset, although not significantly. PER1 is a circadian clock gene involved in $\mathrm{AD}$ [92]. In the CSF, several AD markers were also altered, such as secreted phosphoprotein 1 (SPP1), C$\mathrm{X}-\mathrm{C}$ motif chemokine ligand 10 (CXCL10), and TNF receptor superfamily member $1 \mathrm{~B}$ (TNFRSF1B) (Fig. 3b). TNFR SF1B showed consistent expression changes in $\mathrm{AD}$ or $\mathrm{AD}$ related pathologies, as well as in COVID-19 patient CSF samples. We also found that CXCL10 protein level was increased in CSF of COVID-19 patients [93] (Fig. 3b).

To understand the potential pathological consequences of these alterations by SARS-CoV-2 infection, we interrogated the human protein-protein interactome, the ten HCoVs host factor datasets, and the transcriptome data from PBMCs (Fig. 3c) of COVID-19 patients and CSF samples of neuro-COVID-19 patients (Fig. 3d). We selected three AD blood markers (TGFB1, GSTM3, and NKTR) and three CSF markers (SPP1, CXCL10, and TNFRSF $1 B$ ) as examples. Figure $3 \mathrm{c}$, d shows the PPIs among these markers (centered nodes) and their neighbors, which interact with many DEGs or SARS-CoV-2 host factors. For example, NKTR interacts with zinc finger $\mathrm{CCH}$-type containing 18 (ZC3H18) (SARS-CoV-2 host factor), small nuclear interacting protein 1 (SNIP1) (SARS-CoV-1 and SARS-CoV-2 host factor), and casein kinase II subunit alpha (CSNK2A2) (SARS-CoV-1, SARS-CoV-2, and MERS-CoV host factor). NKTR and its PPI partners transcription initiation factor TFIID subunit 1 (TAF1), 40S ribosomal protein S14 (RPS14), and arrestin beta 2 (ARRB2) are differentially expressed in the PBMCs of COVID-19 patients. ARRB2 inhibits toll- 


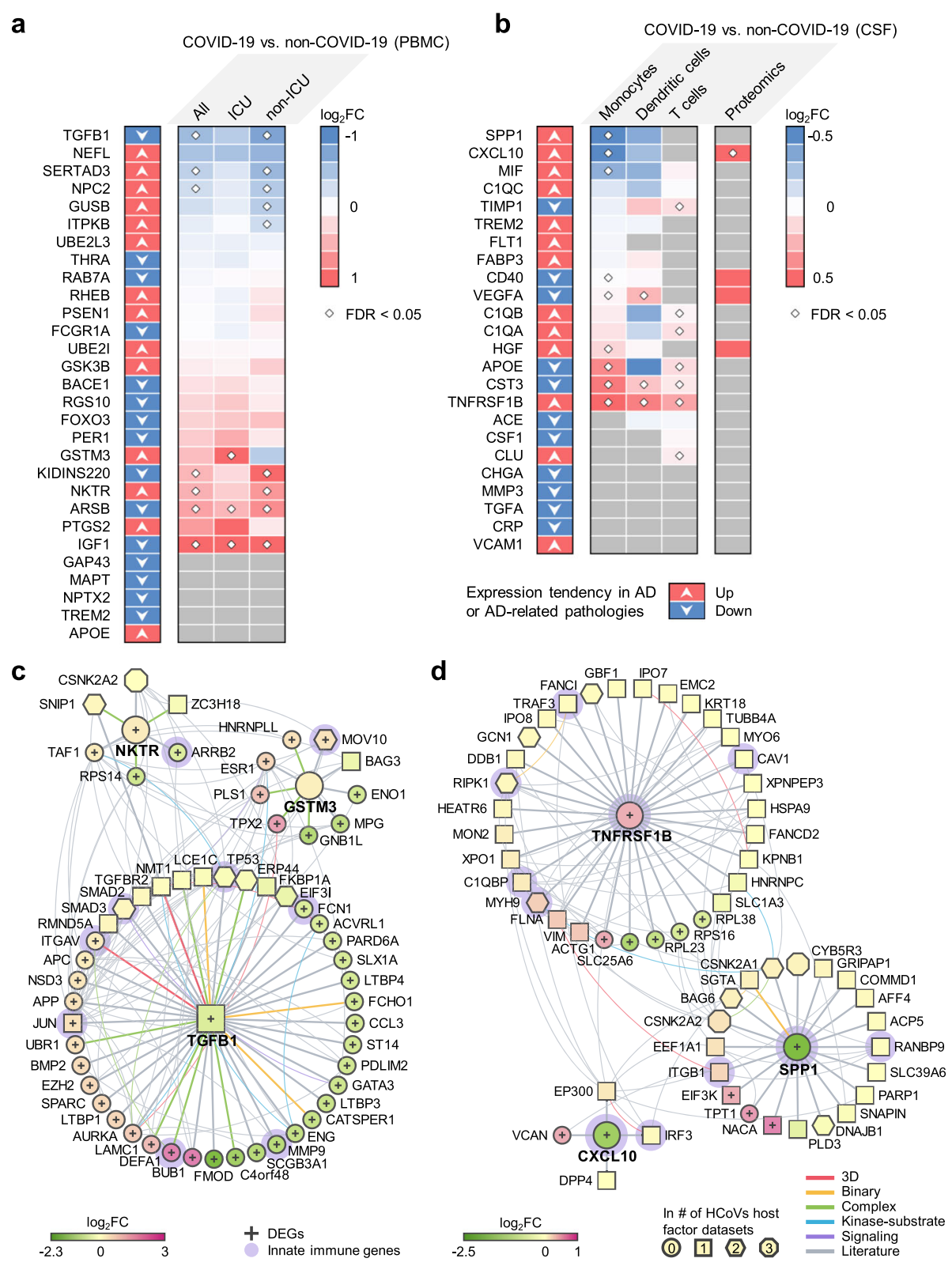

Fig. 3 Neuroinflammation-mediated association between COVID-19 and Alzheimer's disease (AD). The expression of AD a blood and $\mathbf{b}$ cerebrospinal fluids (CSF) protein markers in COVID-19 patients. Heatmaps show the fold change (FC) of the comparisons indicated above. $\mathbf{c}$, $\mathbf{d}$ Network analyses of the AD markers that are differentially expressed in COVID-19 vs. non-COVID-19. Neighbors of these markers that are the SARS-CoV-2 host factors (non-circle nodes) or are DEGs (denoted by " + ") in the COVID-19 datasets are shown. Node shape indicates the number of SARS-CoV-2 host factor datasets that contain the node. Edge colors indicate the protein-protein interaction source type. PBMC, peripheral blood mononuclear cells. DEG, differentially expressed genes. ICU, intensive care unit

like receptor 4 (TLR4)-mediated inflammatory signaling [94], which is activated by the SARS-CoV-2 spike protein [95]. In CSF, innate immune genes SPP1, CXCL10, and TNFRSF1B are differentially expressed in COVID19 vs. non-COVID-19 patients. Many of their PPI partners are also SARS-CoV-2 host factors, among which some are innate immune gene products, such as integrin subunit beta 1 (ITGB1), which is highly expressed in airway epithelial cells [96], and TNF receptor-associated factor 3 (TRAF3), which controls type I interferon (IFNI) production [97]. Integrins may function as an alternative docking receptor for SARS-CoV-2 [98], and ITGB1 is also essential for the migration of monocytes across the endothelium [99].

In summary, the expression of these selected $\mathrm{AD}$ markers was significantly altered by SARS-CoV-2 infection. Using network and multi-omics data analysis, we found that SARS-CoV-2 infection impacts several 
immune-related genes/pathways that could lead to ADlike neurologic impairment.

\section{Elevated expression of SARS-CoV-2 host factors in brain endothelial cells}

We next evaluated the susceptibility of brain endothelial cells to SARS-CoV-2 infection and potential microvascular injury. For this, we analyzed the single-nuclei RNAsequencing dataset from the prefrontal cortex region of $12 \mathrm{AD}$ patients and 9 cognitively healthy controls [51] (Fig. 4a). We examined expression of SARS-CoV-2 entry factors across the six cell types: astrocytes, endothelial cells, excitatory neurons, inhibitory neurons, microglia, and oligodendrocytes (Fig. 4b). We observed low expression levels of $A C E 2$, transmembrane serine protease 2 (TMPRSS2), furin (FURIN), and neuropilin 1 (NRP1) in neurons in both $\mathrm{AD}$ patients and healthy controls. For example, ACE2 and TMPRSS 2 are mostly absent across all six cell types. However, NRP1 is expressed in endothelial cells, astrocytes, and microglia, and expression is elevated in these cell types than in neurons. NRP1 was reported to mediate SARS-CoV-2 cell entry in addition to ACE2 and TMPRSS2 [100, 101]. Basigin $(B S G)$ is much more strongly expressed in endothelial cells than other cell types, and has been reported as a docking receptor for SARS-CoV-2 [102], in addition to ACE2 and NRP1. Among the proteases, FURIN has an elevated expression in endothelial cells compared to other cell types, and cystatin B (CSTB) is highly expressed in microglia. Differential gene expression analysis confirmed that BSG and FURIN have significantly higher expression in the brain endothelial cells than in other cell types (Table S6). In addition to these SARS-CoV-2 entry factors, we also found elevated expression of antiviral defense system genes in brain endothelial cells, including lymphocyte antigen 6 family member $\mathrm{E}$ (LY6E), interferon-induced transmembrane protein 2 (IFITM2) and 3 (IFITM3), and interferon alpha and beta receptor subunit 1 (IFNAR1). These findings are further confirmed in a second single-nuclei RNA-sequencing dataset [52] (Fig. S4). LY6E impairs entry of coronavirus by inhibiting spike protein-mediated membrane fusion [103]. IFN-I receptors (IFNAR) play important roles in IFN-I-mediated antiviral immunity [104], and IFNinduced transmembrane protein 3 (IFITM3) inhibits SARS-CoV-2 cell entry $[105,106]$. IFITM3 is also associated with $\mathrm{AD}$ through its ability to bind and upregulate $\gamma$-secretase, which leads to increased $A \beta$ production [107]. Network analysis also revealed several important PPI partners of these antiviral defense genes (Fig. 4c), such as signal transducer and activator of transcription 3 (STAT3) and janus kinase 1 (JAK1). These immune genes are the HCoVs host factors and have significantly elevated expression in endothelial cells compared to other cell types of the brain. The JAK-STAT signaling pathway mediates the biological functions of several cytokines involved in cytokine release syndrome (CRS) [108], which is common in COVID-19 [109]. Notably, JAK inhibition reduces SARS-CoV-2 infection in the liver and reduces overall morbidity and mortality in COVID-19 patients in a pilot clinical trial [110]. Inhibition of JAK-STAT signaling has therefore been proposed as a treatment strategy for COVID-19 [111].

\section{Reduced expression of antiviral defense genes in $A P O E$ E4/E4 individuals}

It has been suggested that SARS-CoV-2 neurotropism in neurons and astrocytes may be affected by the $A P O E$ genotype [112]. Individuals carrying $A P O E$ E2 have decreased AD risk $[113,114]$, and those carrying APOE E4 have increased risk [114], relative to carriers of the normal APOE E2 allele. Therefore, we examined the expression of these genes in endothelial cells (Fig. 4d) and other cell types (Fig. S5). We found that the expression of some of these genes varies by APOE genotype. NRP1 $\left(\log _{2} \mathrm{FC}=0.52, \mathrm{FDR}=1.00\right)$ and $B S G\left(\log _{2} \mathrm{FC}=0.34\right.$, FDR $=1.00$ ) have slightly elevated expression (lack of statistical significance) in E3/E3 AD patients than in E4/ E4 AD patients in endothelial cells (Table S7). The expression of FURIN and CTSB are similar between APOE $\mathrm{E} 3 / \mathrm{E} 3$ and $\mathrm{E} 4 / \mathrm{E} 4 \mathrm{AD}$ patients $\left(\left|\log _{2} \mathrm{FC}\right|<0.1, \mathrm{FDR}>\right.$ $0.05)$. Yet, several antiviral defense genes, including LY6E, IFITM2, IFITM3, and IFNAR1, have overall elevated expression in E3/E3 AD patients compared to E4/ E4 AD patients (Fig. 4d). These results suggest that $\mathrm{AD}$ patients with $A P O E$ E4/E4 genotype may have less active antiviral defense gene expression activities, which could render them at increased risk for SARS-CoV-2 infection.

\section{Overall low expression of SARS-CoV-2 host factors in human brain}

As SARS-CoV-2 infection depends on key entry factors, including ACE2, TMPRSS2, FURIN, and NRP1, we first examined expression of these entry factors in healthy tissues using GTEx data [77]. We found overall low expression of SARS-CoV-2 entry factors (ACE2, TMPRSS2, FURIN, and NRP1) in the human brain (Fig. S6). Brainspecific expression of the four SARS-CoV-2 entry factors (blue bars in the highlighted yellow column of Fig. 5a) are lower than in other tissues.

It is possible that these entry factors express in certain brain regions, such as thalamus, brain stem, and hippocampus, which may be targeted by SARS-CoV-2 from the olfactory bulb $[115,116]$. Therefore, we further examined expression of these entry factors across different brain regions. Among the 13 brain regions, no region showed high specificity for ACE2, TMPRSS2, FURIN, or NRP1 (Fig. 5b and Fig. S7). The Spearman's rank 


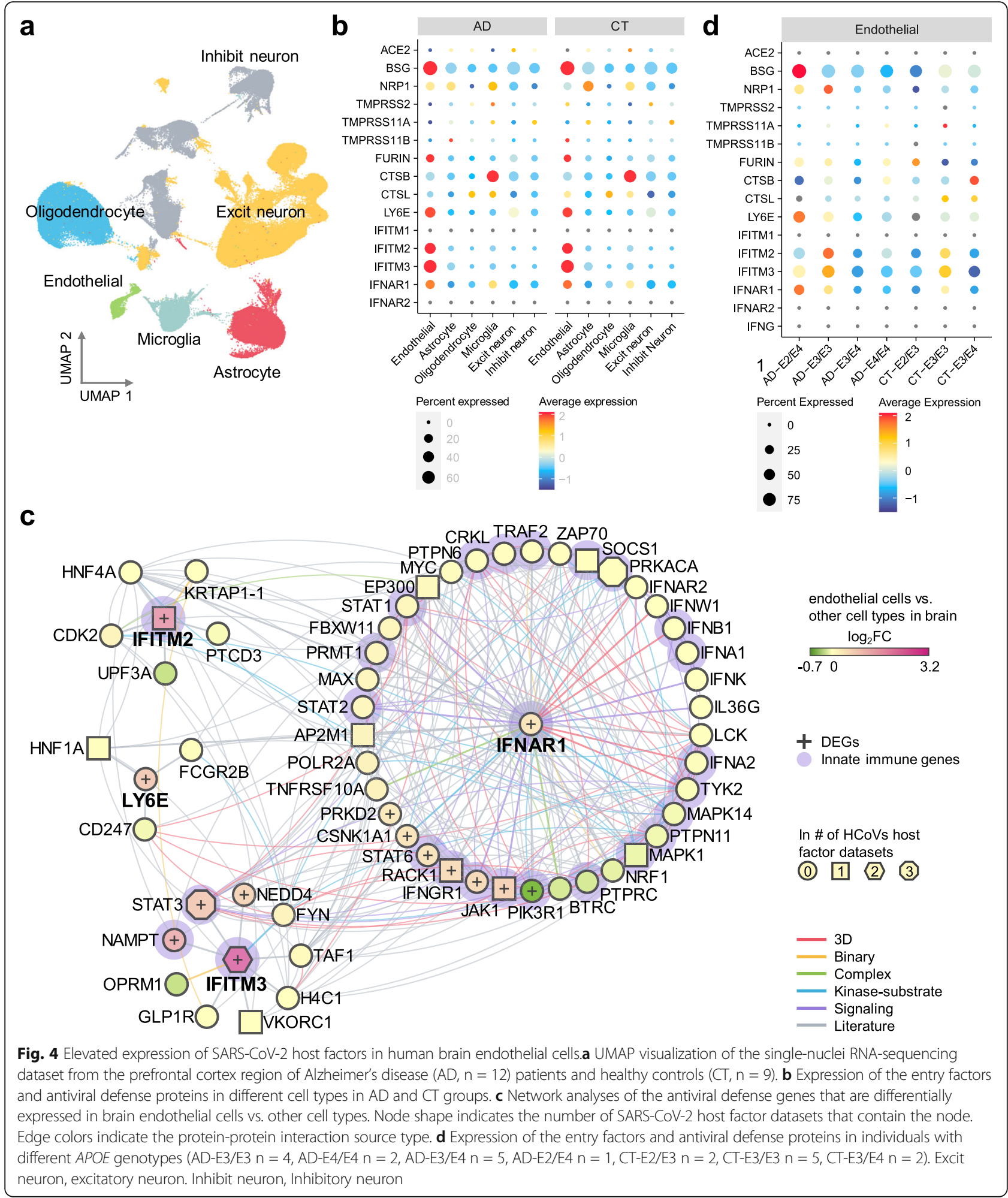

correlation coefficient $(\rho)$ for TMPRSS2, FURIN, and $N R P 1$ with $A C E 2$ does not show a co-expression $\left(|\rho|_{\max }\right.$ $=0.42$ for ACE2 and FURIN in nucleus accumbens) in any of the 13 brain regions (Fig. 5C).
It has been reported that $A C E 2$ has an overall low expression in lung $[117,118]$, as also shown in Fig. 5a, but higher expression in certain cell types such as lung alveolar type II (AT2) epithelial cells [117], bronchial 

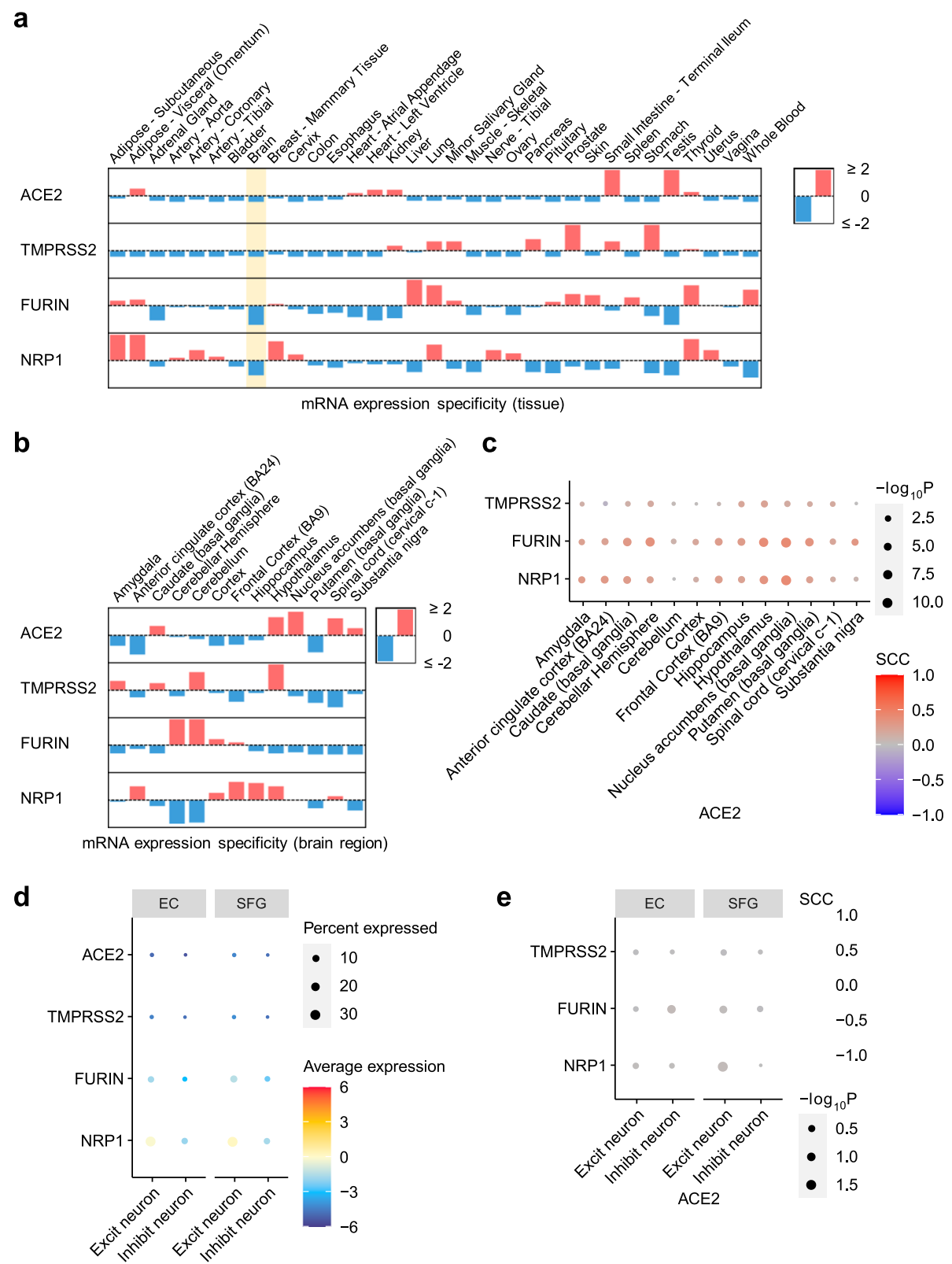

Fig. 5 Expression of key SARS-CoV-2 entry factors across 33 human tissues, 13 brain regions, and brain cell types/subpopulations. a Expression specificity of key SARS-CoV-2 entry factors in 33 tissues and $\mathbf{b}$ expression specificity of these genes in 13 brain regions using data from the GTEx database (see the "Materials and methods" section). c Co-expression of TMPRSS2, FURIN, and NRP1 Vs. ACE2 in the brain regions. $\mathbf{d}$ Expression of key SARS-CoV-2 entry factors in the neuron cells. e Co-expression of TMPRSS2, FURIN, and NRP1 vs. ACE2 in the neuron. SCC, Spearman's rank correlation coefficient. EC, entorhinal cortex. SFG, superior frontal gyrus. Excit neuron, excitatory neuron. Inhibit neuron, Inhibitory neuron

secretory cells [119], nasal mucosa [118], and absorptive enterocytes in the ileum [120]. This prompted us to investigate the brain expression of the entry factors at the single-cell/nuclei level. Using single-nuclei RNAsequencing data of the caudal entorhinal cortex and the superior frontal gyrus from AD patients [50], we examined the expression of the four key SARS-CoV-2 entry factors in the excitatory neuron and inhibitory neuron cells (Fig. 5d). Notably, we found very low expression of SARS-CoV-2 entry factors as well, consistent with our findings shown in Fig. 4b. In addition, co-expression of TMPRSS2, FURIN, or NRP1 with ACE2 is low (Fig. 5e, $|\rho|_{\max }=0.03$ for $A C E 2$ and FURIN in inhibitory neurons in the entorhinal cortex region). These results suggest that neurons are unlikely to be a direct target for SARSCoV-2 infection. However, we should note that even 
though its expression is low overall, NRP1 has a relatively higher expression than the other three genes. Together, these expression results at the tissue, brain region, and single-nuclei levels suggest that SARS-CoV2 is unlikely to directly invade brain and that cognitive impairment with COVID-19 is more likely caused by neuroinflammation (Fig. 3) and microvascular injury (Fig. 4).

\section{Discussion}

The negative effects of COVID-19 on the CNS may have a long-term impact that could possibly increase the likelihood of developing AD-like dementia [1, 2, 4, 5, 121]. Here, we investigated the potential mechanisms for this effect. Using network proximity measure in the human PPI, we found strong network-based relationship between SARS-CoV-2 host factors (based on PPI assays and CRISPR-Cas9 genetic assays) and disease-associated genes/proteins of dementia-like cognitive impairment. Network analysis of the SARS-CoV-2 host factors and $\mathrm{AD}$-associated genes/proteins reveals that these two sets have significant network proximities in the human interactome. Several AD-associated proteins were highlighted, including RAB7A, TGFB1, and VCAM1, with potentially high impact on the network according to their degrees and eigenvector centralities. In addition, the expression of these genes is also altered in COVID19 patients based on the results of transcriptomic analyses.

Previous studies have shown that SARS-CoV-2 is absent from the brain [22] and CSF [13]. However, evidence also exists that SARS-CoV-2 may directly infect the brain [19-21]. To test the possibility of direct brain invasion by SARS-CoV-2, we investigated the expression of key entry factors of SARS-CoV-2 at three levels: tissue, brain regions, and brain cell types. We found very low expression of ACE2 and TMPRSS2 in the brain and neurons. ACE2 is the main known SARS-CoV-2 docking receptor [117-119]; yet, it has little to no expression in neurons (Figs. 4b and 5d). Recent studies found two additional SARS-CoV-2 docking receptors, NRP1 [100, 101] and BSG [102]. BSG, NRP1, and FURIN have elevated expression in the endothelial cells in the prefrontal cortex region of both $\mathrm{AD}$ patients and healthy controls compared to other brain cell types (Fig. 4b). Our results suggest that it is unlikely for SARS-CoV-2 to target neurons directly via ACE2. However, we cannot rule out the possibility that SARS-CoV-2 may enter the brain through the cerebral endothelium using receptors such as BSG and NRP1 or other unknown entry factors. In addition, other HCoVs, including HCoV-229E and $\mathrm{HCoV}-\mathrm{OC} 43$, have been detected in human brains [122].

Neuroinflammation is a major hallmark of $\mathrm{AD}$, and we analyzed the expression of $\mathrm{AD}$ blood and CSF markers in PBMCs and CSF of COVID-19 patients. We identified several $\mathrm{AD}$ marker genes (e.g., NKTR, GSTM3, TGFB1, TNFRSF1B, SPP1, and CXCL10) which may provide insights into the shared pathobiology of cognitive dysfunction in COVID-19 and AD. These genes were significantly altered in PBMCs or CSF of COVID-19 patients. Network analysis showed that these genes are enriched in PPIs of immune-related gene products, such as ITGB1 and ARRB2. Moreover, many of the PPI partners of these genes are either the host factors of SARS-CoV-2, or are significantly altered in COVID-19 patients, or both. In addition, the endothelial cells also have elevated expression of antiviral defense genes (LY6E, IFITM2, IFITM3, and IFNAR1) (Fig. 4b). We identified important PPI partners (STAT3 and JAK1) of these genes using network analysis combined with SARS-CoV-2 host factor datasets and differential expression analyses. Due to the inflammation role of the JAKSTAT signaling pathway in COVID-19, its inhibition by baricitinib has been studied as a potential treatment [111] in several clinical trials (NCT04320277 and NCT04321993). We also found that individuals with $A P O E$ E4/E4 have overall lower expression of antiviral defense genes compared to individuals with $A P O E$ E3/ E3, suggesting a lack of expression of these genes and potentially an elevated risk of SARS-CoV-2 infection. Human-induced pluripotent stem cell models showed an elevated susceptibility to SARS-CoV-2 infection in APOE E4/E4 brain cells [112]. Further observations of $A P O E$ related susceptibility to SARS-CoV-2 infection are warranted.

In summary, our observations provide mechanistic insights into two questions: (a) whether SARS-CoV-2 infection could potentially increase the risk of $A D$ and $\mathrm{AD}$-like dementia; and (b) whether individuals with $\mathrm{AD}$ and AD-like dementia have increased risk of SARS$\mathrm{CoV}-2$ infection. Our analyses show a low possibility of direct brain invasion by SARS-CoV-2 (Fig. 5). However, we found significant mechanistic overlap between $\mathrm{AD}$ and COVID-19 (Fig. 2) centered on neuroinflammation and microvascular injury pathways or processes (Figs. 3 and 4). It was found that dementia patients had twice the risk of COVID-19 compared to those without dementia [9]. Although nursing home stays were adjusted in this study [9], it could still potentially explain the high risk in dementia patients, due to a higher nursing home stay tendency in these patients. Other factors, such as aging, a major risk factor of SARS-CoV-2 infection, may also confound the results. We found that the SARSCoV-2 entry factors and the antiviral defense genes have similar transcriptomic expression in the brain cells between $\mathrm{AD}$ patients and control individuals (Fig. 4b and Fig. S4, Table S8). These observations do not suggest an elevated risk of COVID-19 in AD patients by differential 
expression profiles of SARS-CoV-2 entry factors or antiviral defense genes. However, there may be yet unknown SARS-CoV-2 entry factors and antiviral defense systems involving other genes that may have altered expression in $\mathrm{AD}$ patients, which could lead to an elevated risk of COVID-19. Therefore, longitudinal clinical and functional studies are warranted to inspect the causal relationship of dementia and an elevated risk of SARS-CoV-2 infection in the near future.

Due to the shared pathways and network-based relationships between COVID-19 and other diseases such as $\mathrm{AD}$, repurposing COVID-19 treatments may help individuals with other diseases (including $\mathrm{AD}$ ) as well. For example, we recently identified melatonin as a repurposable drug for COVID-19 [38]. Multiple preclinical studies showed that melatonin was a potential treatment for $\mathrm{AD}$ as well $[123,124]$. The methodologies utilizing omics data and unbiased network-based analysis in this study can be applied to other infectious diseases based on the high generalizability of network proximity measure $[33,38,56]$. Our future works include investigating the causal relationships using techniques such as Mendelian randomization analysis and providing visualization and analyses tools in a web server similar to our recent work [42].

\section{Limitations}

We acknowledge several limitations. First, our human protein-protein interactome was built using high-quality data from multiple sources; yet it is still incomplete. The PPIs in our interactome are undirected. However, it has been shown that incorporating the directionality of the human PPI does not change network proximity results [125]. The network associations could be either positive or negative and require further investigation. In addition, as our network proximity analysis relies on diseaseassociated genes, literature bias could affect the results because more highly-studied genes are more likely to appear in the dataset. Highly studied genes, such as innate immune genes, tend to have higher degrees in the human interactome (Fig. S8). Therefore, degree-controlled permutation tests were based on gene sets that had similar degree distributions to the gene sets-of-interest to reduce the effect of literature-based degree biases based on our previous studies [33, 38, 56]. Second, we analyzed expression levels of the key SARS-CoV-2 entry factors and found low expression levels for ACE2 and TMPR SS2. However, we cannot rule out the possibility of SARS-CoV-2 directly targeting the brain via as-yet unidentified mechanisms. Third, although we found several $\mathrm{AD}$ protein markers that have similar alterations in COVID-19 and AD, there are also protein markers that have opposite directions in the gene expression change in $\mathrm{AD}$ and COVID-19. There are several potential explanations, such as small sample size during differential expression analysis, patient heterogeneity in the omics profiling studies, and discrepancy between mRNA and protein expression levels [126]. Possible pathways of neuroinflammation and microvascular injury were tested using data of either individuals with AD or COVID-19, but not both. Future studies using genetics and multi-omics data from individuals with both $\mathrm{AD}$ and COVID-19 will be needed to confirm and extend these network-based findings. In addition, microvasculature components other than endothelial cells, such as pericytes, are not investigated in this study. Fourth, the significance of our findings in the context of the general population of COVID-19 frequently suffering from "brain fog" without a formal diagnosis of $\mathrm{AD}$ needs further investigation. Last, the potential mechanisms of key genes and pathways discovered in this study help understand the relations between COVID-19 and its neurological manifestations. However, further clinical and functional observations are needed to determine the causal relationships, such as through the use of Mendelian randomization.

\section{Conclusions}

In this study, we investigated COVID-19-assoicated neurological manifestations using both network medicine methodologies and bulk/single-cell/single-nuclei transcriptomic data analyses. We identified strong shared neuroinflammatory responses between COVID19 and AD. Several AD markers (CXCL10, TNFRSF1B, SPP1, TGFB1, GSTM3, and NKTR) have significantly altered expression in COVID-19 patients. Low expression levels of SARS-CoV-2 entry factors were found in human brains, indicating low possibility of direct brain damage by the virus. Transcriptomic analyses showed elevated expression levels of SARS-CoV-2 host factors (BSG and FURIN) and antiviral defense genes (LY6E, IFITM2, IFITM3, and IFNAR1) in brain endothelial cells compared to other cell types, suggesting possible brain microvascular injury by SARS-CoV-2 infection. In addition, individuals with $A P O E \mathrm{E} 4 / \mathrm{E} 4$ may have increased risk of SARS-CoV-2 infection by an overall lower expression of antiviral defense genes (LY6E, IFIT M2, IFITM3, and IFNAR1) compared to individuals with $A P O E$ E3/E3. Altogether, these results can improve our understanding of COVID-19-associated neurological manifestations and provide guidance for future risk management of potential cognitive impairment by SARS-CoV-2 infection. Our findings could lay the foundation for future research that ultimately leads to testable and measurable serum biomarkers that could identify patients at highest risk of neurological complications with COVID-19. 


\section{Supplementary Information}

The online version contains supplementary material available at https://doi. org/10.1186/s13195-021-00850-3.

Additional file 1: Figure S1. Functional enrichment analysis and largest connected component of the six CRISPR-Cas9-based SARS-CoV-2 host factor datasets. Figure S2. Network proximity results using different numbers of top genes from the CRISPR-Cas9-based SARS-CoV-2 host factor datasets. Figure S3. Single-cell level expression of AD blood markers in the PBMC samples of COVID-19 patients. Figure S4. Expression spectrum of the SARS-CoV-2 entry factors in the entorhinal cortex from Alzheimer's disease patients and controls. Figure S5. Expression spectrum of the SARS-CoV-2 entry factors in individuals with different APOE genotypes. Figure S6. Expression of the key SARS-CoV-2 entry factors in different tissues. Figure S7. Expression of the key SARS-CoV-2 entry factors in different brain regions. Figure S8. Cumulative degree distribution of 964 innate immune genes, 14267 brain expressed genes, and 3383 brain specific genes.

Additional file 2: Table S1. SARS-CoV-2 host factor datasets. Additional file 3: Table S2. Neurological diseases-associated genes/ proteins.

Additional file 4: Table S3. Alzheimer's disease markers and their expressions.

Additional file 5: Table S4. Transcriptomic datasets used in this study. Additional file 6: Table S5. Raw data and network analysis results of the nodes in Fig. $2 b$.

Additional file 7: Table S6. Differentially expressed genes in brain endothelial cells vs. other cell types.

Additional file 8: Table S7. Differentially expressed genes in brain endothelial cells by comparing APOE genotype E3/E3 and E4/E4 in Alzheimer's disease patients.

Additional file 9: Table S8. Differentially expressed genes in Alzheimer's disease patients vs. normal controls for each cell type.

\section{Acknowledgements}

We thank all helpful discussions and critical comments regarding this manuscript from the COVID-19 Research Intervention Advisory Committee members at the Cleveland Clinic.

\section{Authors' contributions}

F.C. conceived the study. Y.Z., J.X., and Y.H. performed data processing and analyses. A.K., R.M., H.Y., Y.L., J.B.L., A.A.P., and L.J. discussed and interpreted all results. Y.Z. and F.C. wrote and critically revised the manuscript and all authors gave final approval.

\section{Funding}

This work was supported by the National Institute of Aging (R01AG066707 and 3R01AG066707-01S1) and the National Heart, Lung, and Blood Institute (R00HL138272) to F.C. This work has also been supported by the National Institute of Neurological Disorders and Stroke (3R01NS097719-04S1) to F.C. and L.J. This work has also been supported in part by the VeloSano Pilot Program (Cleveland Clinic Taussig Cancer Institute) to F.C.

\section{Availability of data and materials}

The transcriptomic datasets used in this study (GSE147528, GSE157827, GSE138852, GSE157103, GSE149689, and GSE163005) were downloaded from the NCBI GEO database (https://www.ncbi.nlm.nih.gov/geo/). The GTEx v8 dataset was downloaded from https://gtexportal.org/home/. The human protein-protein interactome and the network proximity code can be found in https://github.com/ChengF-Lab/COVID-19_Map. Interactive version of Fig. $2 \mathrm{~b}$ and all original network files can be found in https://github.com/ChengFLab/COVID-19_Brain. The AD multi-omics datasets can be found in https:// alzgps.lerner.ccf.org/.

\section{Declarations}

Ethics approval and consent to participate

Not applicable

\section{Consent for publication}

Not applicable

\section{Competing interests}

Dr. Leverenz has received consulting fees from Acadia, Biogen, Eisai, GE Healthcare, and Sunovion. Dr. Cheng has received consulting fees from the National Institute of Aging. The other authors declare that they have no competing interests.

\section{Author details}

'Genomic Medicine Institute, Lerner Research Institute, Cleveland Clinic, Cleveland, OH 44195, USA. '2Department of Molecular Medicine, Cleveland Clinic Lerner College of Medicine, Case Western Reserve University, Cleveland, OH 44195, USA. ${ }^{3}$ Lou Ruvo Center for Brain Health, Neurological Institute, Cleveland Clinic, Cleveland, OH 44195, USA. ${ }^{4}$ Neurological Institute, Cleveland Clinic, Cleveland, OH 44195, USA. ${ }^{5}$ Department of Medical and Molecular Genetics, Indiana University School of Medicine, Indianapolis, IN 46202, USA. ${ }^{6}$ Weill Institute for Cell and Molecular Biology, Cornell University, Ithaca, NY 14850, USA. ${ }^{7}$ Department of Computational Biology, Cornell University, Ithaca, NY 14850, USA. ${ }^{8}$ Tri-Institutional Training Program in Computational Biology and Medicine, Cornell University, Ithaca, NY 14850, USA. ${ }^{9}$ Harrington Discovery Institute, University Hospitals Cleveland Medical Center, Cleveland, $\mathrm{OH}$ 44106, USA. ${ }^{10}$ Department of Psychiatry, Case Western Reserve University, Cleveland, OH 44106, USA. ${ }^{11}$ Geriatric Psychiatry, GRECC, Louis Stokes Cleveland VA Medical Center, Cleveland, OH 44106, USA. ${ }^{12}$ Institute for Transformative Molecular Medicine, School of Medicine, Case Western Reserve University, Cleveland, OH 44106, USA. ${ }^{13}$ Weill Cornell Autism Research Program, Weill Cornell Medicine of Cornell University, New York, NY 10065, USA. ${ }^{14}$ Department of Neuroscience, School of Medicine, Case Western Reserve University, Cleveland, $\mathrm{OH} 44106$, USA. ${ }^{15}$ Case Comprehensive Cancer Center, School of Medicine, Case Western Reserve University, Cleveland, $\mathrm{OH}$ 44106, USA.

Received: 13 March 2021 Accepted: 28 May 2021

Published online: 09 June 2021

\section{References}

1. Mao L, Jin H, Wang M, Hu Y, Chen S, He Q, et al. Neurologic Manifestations of Hospitalized Patients With Coronavirus Disease 2019 in Wuhan, China. JAMA Neurol. 2020;77(6):683-90. https://doi.org/10.1001/jamaneurol.202 0.1127 pmid: 32275288.

2. Li YC, Bai WZ, Hashikawa T. The neuroinvasive potential of SARS-CoV2 may play a role in the respiratory failure of COVID-19 patients. J Med Virol. 2020; 92(6):552-5. https://doi.org/10.1002/jmv.25728 pmid: 32104915.

3. Rogers JP, Chesney E, Oliver D, Pollak TA, McGuire P, Fusar-Poli P, et al. Psychiatric and neuropsychiatric presentations associated with severe coronavirus infections: a systematic review and meta-analysis with comparison to the COVID-19 pandemic. Lancet Psychiatry. 2020;7(7):611-27. https://doi.org/10.1016/S2215-0366(20)30203-0 pmid: 32437679.

4. Zhou H, Lu S, Chen J, Wei N, Wang D, Lyu H, et al. The landscape of cognitive function in recovered COVID-19 patients. J Psychiatr Res. 2020;129: 98-102. https://doi.org/10.1016/j.jpsychires.2020.06.022 pmid: 32912598.

5. Miners S, Kehoe PG, Love S. Cognitive impact of COVID-19: looking beyond the short term. Alzheimers Res Ther. 2020;12(1):170. https://doi.org/10.1186/ s13195-020-00744-w pmid: 33380345.

6. Romero-Sanchez CM, Diaz-Maroto I, Fernandez-Diaz E, Sanchez-Larsen A, Layos-Romero A, Garcia-Garcia J, et al. Neurologic manifestations in hospitalized patients with COVID-19: The ALBACOVID registry. Neurology. 2020;95(8):e1060-e70. https://doi.org/10.1212/WNL.0000000000009937 pmid: 32482845 .

7. Meppiel E, Peiffer-Smadja N, Maury A, Bekri I, Delorme C, Desestret V, et al. Neurologic manifestations associated with COVID-19: a multicentre registry. Clin Microbiol Infect. 2021;27(3):458-66. https://doi.org/10.1016/j.cmi.202 0.11 .005 pmid: 33189873.

8. Taquet M, Geddes JR, Husain M, Luciano S, Harrison PJ. 6-month neurological and psychiatric outcomes in 236379 survivors of COVID-19: a 
retrospective cohort study using electronic health records. Lancet Psychiatry. 2021;8(5):416-27. https://doi.org/10.1016/S2215-0366(21)00084-5 pmid: 33836148

9. Wang Q, Davis PB, Gurney ME, Xu R. COVID-19 and dementia: Analyses of risk, disparity, and outcomes from electronic health records in the US. Alzheimers Dement. 2021. https://doi.org/10.1002/alz.12296 pmid: 33559975.

10. Atkins JL, Masoli JAH, Delgado J, Pilling LC, Kuo CL, Kuchel GA, et al. Preexisting Comorbidities Predicting COVID-19 and Mortality in the UK Biobank Community Cohort. J Gerontol A Biol Sci Med Sci. 2020;75(11): 2224-30. https://doi.org/10.1093/gerona/glaa183 pmid: 32687551.

11. Bianchetti A, Rozzini R, Guerini F, Boffelli S, Ranieri P, Minelli G, et al. Clinical Presentation of COVID19 in Dementia Patients. J Nutr Health Aging. 2020; 24(6):560-2. https://doi.org/10.1007/s12603-020-1389-1 pmid: 32510106.

12. Beaud V, Crottaz-Herbette S, Dunet V, Vaucher J, Bernard-Valnet R, Du Pasquier R, et al. Pattern of cognitive deficits in severe COVID-19. J Neurol Neurosurg Psychiatry. 2021;92(5):567-8. https://doi.org/10.1136/jnnp-2020-32 5173 pmid: 33219042

13. Helms J, Kremer S, Merdji H, Clere-Jehl R, Schenck M, Kummerlen C, et al. Neurologic Features in Severe SARS-CoV-2 Infection. N Engl J Med. 2020; 382(23):2268-70. https://doi.org/10.1056/NEJMc2008597 pmid: 32294339.

14. Helms J, Kremer S, Merdji H, Schenck M, Severac F, Clere-Jehl R, et al. Delirium and encephalopathy in severe COVID-19: a cohort analysis of ICU patients. Crit Care. 2020;24(1):491. https://doi.org/10.1186/s13054-020-032 00-1 pmid: 32771053.

15. Al-Aly Z, Xie Y, Bowe B. High-dimensional characterization of post-acute sequalae of COVID-19. Nature. 2021. https://doi.org/10.1038/s41586-021-03 553-9 pmid: 33887749.

16. Jaywant A, Vanderlind WM, Alexopoulos GS, Fridman CB, Perlis RH, Gunning FM. Frequency and profile of objective cognitive deficits in hospitalized patients recovering from COVID-19. Neuropsychopharmacology. 2021. https://doi.org/10.1038/s41386-021-00978-8 pmid: 33589778.

17. Janbek J, Frimodt-Møller N, Laursen TM, Waldemar G. Dementia identified as a risk factor for infection-related hospital contacts in a national, population-based and longitudinal matched-cohort study. Nature Aging 2021;1(2):226-33. https://doi.org/10.1038/s43587-020-00024-0.

18. ladecola C, Anrather J, Kamel H. Effects of COVID-19 on the Nervous System. Cell. 2020;183(1):16-27. https://doi.org/10.1016/j.cell.2020.08.028 e1. pmid: 32882182.

19. Meinhardt J, Radke J, Dittmayer C, Franz J, Thomas C, Mothes R, et al. Olfactory transmucosal SARS-CoV-2 invasion as a port of central nervous system entry in individuals with COVID-19. Nat Neurosci. 2021;24(2):168-75. https://doi.org/10.1038/s41593-020-00758-5 pmid: 33257876.

20. Matschke J, Lutgehetmann M, Hagel C, Sperhake JP, Schroder AS, Edler C, et al. Neuropathology of patients with COVID-19 in Germany: a postmortem case series. Lancet Neurol. 2020;19(11):919-29. https://doi.org/10.1 016/S1474-4422(20)30308-2 pmid: 33031735

21. Song E, Zhang C, Israelow B, Lu-Culligan A, Prado AV, Skriabine S, et al. Neuroinvasion of SARS-CoV-2 in human and mouse brain. J Exp Med. 2021; 218(3). https://doi.org/10.1084/jem.20202135 pmid: 33433624

22. Lee MH, Perl DP, Nair G, Li W, Maric D, Murray H, et al. Microvascular Injury in the Brains of Patients with Covid-19. N Engl J Med. 2021;384(5):481-3. https://doi.org/10.1056/NEJMc2033369 pmid: 33378608.

23. de Erausquin GA, Snyder H, Carrillo M, Hosseini AA, Brugha TS, Seshadri S, et al. The chronic neuropsychiatric sequelae of COVID-19: The need for a prospective study of viral impact on brain functioning. Alzheimers Dement. 2021. https://doi.org/10.1002/alz.12255 pmid: 33399270

24. Rhea EM, Logsdon AF, Hansen KM, Williams LM, Reed MJ, Baumann KK, et al. The S1 protein of SARS-CoV-2 crosses the blood-brain barrier in mice. Nat Neurosci. 2020;24(3):368-78. https://doi.org/10.1038/s41593-020-007718. pmid: 33328624

25. Buzhdygan TP, DeOre BJ, Baldwin-Leclair A, Bullock TA, McGary HM, Khan JA, et al. The SARS-CoV-2 spike protein alters barrier function in 2D static and 3D microfluidic in-vitro models of the human blood-brain barrier. Neurobiol Dis. 2020;146:105131. https://doi.org/10.1016/j.nbd.2020.105131 pmid: 33053430

26. Blanco-Melo D, Nilsson-Payant BE, Liu WC, Uhl S, Hoagland D, Moller R, et al. Imbalanced Host Response to SARS-CoV-2 Drives Development of COVID-19. Cell. 2020;181(5):1036-45. https://doi.org/10.1016/j.cell.2020.04.026 e9. pmid: 32416070

27. Bojkova D, Klann K, Koch B, Widera M, Krause D, Ciesek S, et al. Proteomics of SARS-CoV-2-infected host cells reveals therapy targets. Nature. 2020;
583(7816):469-72. https://doi.org/10.1038/s41586-020-2332-7 pmid: 32408336

28. Gordon DE, Jang GM, Bouhaddou M, Xu J, Obernier K, White KM, et al. A SARS-CoV-2 protein interaction map reveals targets for drug repurposing. Nature. 2020;583(7816):459-68. https://doi.org/10.1038/s41586-020-2286-9 pmid: 32353859.

29. Gordon DE, Hiatt J, Bouhaddou M, Rezelj W, Ulferts S, Braberg H, et al. Comparative host-coronavirus protein interaction networks reveal pan-viral disease mechanisms. Science. 2020;370(6521)). https://doi.org/10.1126/ science.abe9403 pmid: 33060197

30. Daniloski Z, Jordan TX, Wessels HH, Hoagland DA, Kasela S, Legut M, et al. Identification of Required Host Factors for SARS-CoV-2 Infection in Human Cells. Cell. 2021;184(1):92-105. https://doi.org/10.1016/j.cell.2020.10.030 e16. pmid: 33147445

31. Wang R, Simoneau CR, Kulsuptrakul J, Bouhaddou M, Travisano KA, Hayashi JM, et al. Genetic Screens Identify Host Factors for SARS-CoV-2 and Common Cold Coronaviruses. Cell. 2021;184(1):106-19. https://doi.org/10.1 016/j.cell.2020.12.004 e14. pmid: 33333024.

32. Wei J, Alfajaro MM, DeWeirdt PC, Hanna RE, Lu-Culligan WJ, Cai WL, et al. Genome-wide CRISPR Screens Reveal Host Factors Critical for SARS-CoV-2 Infection. Cell. 2021;184(1):76-91. https://doi.org/10.1016/j.cell.2020.10.028 e13. pmid: 33147444.

33. Cheng F, Desai RJ, Handy DE, Wang R, Schneeweiss S, Barabasi AL, et al. Network-based approach to prediction and population-based validation of in silico drug repurposing. Nat Commun. 2018;9(1):2691. https://doi.org/10.1 038/s41467-018-05116-5 pmid: 30002366.

34. Cheng F, Lu W, Liu C, Fang J, Hou Y, Handy DE, et al. A genome-wide positioning systems network algorithm for in silico drug repurposing. Nat Commun. 2019;10(1):3476. https://doi.org/10.1038/s41467-019-10744-6 pmid: 31375661

35. Fang J, Pieper AA, Nussinov R, Lee G, Bekris L, Leverenz JB, Cummings J, Cheng $\mathrm{F}$ Harnessing endophenotypes and network medicine for Alzheimer's drug repurposing. Med Res Rev. 2020;40(6):2386-2426. doi: https://doi.org/10.1002/med.21709. pmid: 32656864

36. Zhou Y, Wang F, Tang J, Nussinov R, Cheng F. Artificial intelligence in COVID-19 drug repurposing. Lancet Digit Health. 2020;2(12):e667-e76. https://doi.org/10.1016/S2589-7500(20)30192-8 pmid: 32984792.

37. Zhou Y, Hou Y, Shen J, Huang Y, Martin W, Cheng F. Network-based drug repurposing for novel coronavirus 2019-nCoV/SARS-CoV-2. Cell Discov. 2020;6(1):14. https://doi.org/10.1038/s41421-020-0153-3. pmid: 32194980.

38. Zhou Y, Hou Y, Shen J, Mehra R, Kallianpur A, Culver DA, et al. A network medicine approach to investigation and population-based validation of disease manifestations and drug repurposing for COVID-19. PLOS Biol. 2020; 18(11):e3000970. https://doi.org/10.1371/journal.pbio.3000970 pmid: 33156843

39. Kuleshov MV, Jones MR, Rouillard AD, Fernandez NF, Duan Q, Wang Z, et al. Enrichr: a comprehensive gene set enrichment analysis web server 2016 update. Nucleic Acids Res. 2016;44(W1):W90-7. https://doi.org/10.1093/nar/ gkw377 pmid: 27141961

40. Stenson PD, Ball EV, Mort M, Phillips AD, Shiel JA, Thomas NS, et al. Human Gene Mutation Database (HGMD): 2003 update. Hum Mutat. 2003;21(6):57781. https://doi.org/10.1002/humu.10212 pmid: 12754702.

41. Bello SM, Shimoyama M, Mitraka E, Laulederkind SJF, Smith CL, Eppig JT, et al. Disease Ontology: improving and unifying disease annotations across species. Dis Model Mech. 2018;11(3):dmm032839. https://doi.org/10.1242/ dmm.032839 pmid: 29590633.

42. Zhou Y, Fang J, Bekris LM, Kim YH, Pieper AA, Leverenz JB, et al. AlzGPS: a genome-wide positioning systems platform to catalyze multi-omics for Alzheimer's drug discovery. Alzheimers Res Ther. 2021;13(1):24. https://doi. org/10.1186/s13195-020-00760-w pmid: 33441136

43. Fang J, Zhang P, Wang Q, Zhou Y, Chiang WC, Cheng R, et al. Networkbased Translation of GWAS Findings to Pathobiology and Drug Repurposing for Alzheimer's Disease. medRxiv. 2020:2020.01.15.20017160. https://doi.org/10.1101/2020.01.15.20017160.

44. Wang $\mathrm{Q}$, Chen $\mathrm{R}$, Cheng $\mathrm{F}$, Wei $\mathrm{O}$, Ji $\mathrm{Y}$, Yang $\mathrm{H}$, et al. A Bayesian framework that integrates multi-omics data and gene networks predicts risk genes from schizophrenia GWAS data. Nat Neurosci. 2019;22(5):691-9. https://doi. org/10.1038/s41593-019-0382-7 pmid: 30988527.

45. Brosseron F, Traschutz A, Widmann CN, Kummer MP, Tacik P, Santarelli F, et al. Characterization and clinical use of inflammatory cerebrospinal fluid 
protein markers in Alzheimer's disease. Alzheimers Res Ther. 2018;10(1):25. https://doi.org/10.1186/s13195-018-0353-3 pmid: 29482610.

46. Meyer PF, Savard M, Poirier J, Morgan D, Breitner J. Alzheimer's Disease Neuroimaging I. Hypothesis: cerebrospinal fluid protein markers suggest a pathway toward symptomatic resilience to AD pathology. Alzheimers Dement. 2019;15(9):1160-71. https://doi.org/10.1016/j.jalz.2019.05.007 pmid: 31405825.

47. Niculescu AB, Le-Niculescu H, Roseberry K, Wang S, Hart J, Kaur A, et al. Blood biomarkers for memory: toward early detection of risk for Alzheimer disease, pharmacogenomics, and repurposed drugs. Mol Psychiatry. 2020; 25(8):1651-72. https://doi.org/10.1038/s41380-019-0602-2 pmid: 31792364.

48. Butler A, Hoffman P, Smibert P, Papalexi E, Satija R. Integrating single-cell transcriptomic data across different conditions, technologies, and species. Nat Biotechnol. 2018;36(5):411-20. https://doi.org/10.1038/nbt.4096 pmid: 29608179.

49. Robinson MD, McCarthy DJ, Smyth GK. edgeR: a Bioconductor package for differential expression analysis of digital gene expression data. Bioinformatics. 2010;26(1):139-40. https://doi.org/10.1093/bioinformatics/ btp616 pmid: 19910308.

50. Leng K, Li E, Eser R, Piergies A, Sit R, Tan M, et al. Molecular characterization of selectively vulnerable neurons in Alzheimer's disease. Nat Neurosci. 2021; 24(2):276-87. https://doi.org/10.1038/s41593-020-00764-7 pmid: 33432193.

51. Lau SF, Cao H, Fu AKY, Ip NY. Single-nucleus transcriptome analysis reveals dysregulation of angiogenic endothelial cells and neuroprotective glia in Alzheimer's disease. Proc Natl Acad Sci U S A. 2020;117(41):25800-9. https:// doi.org/10.1073/pnas.2008762117 pmid: 32989152.

52. Grubman A, Chew G, Ouyang JF, Sun G, Choo XY, McLean C, et al. A singlecell atlas of entorhinal cortex from individuals with Alzheimer's disease reveals cell-type-specific gene expression regulation. Nat Neurosci. 2019; 22(12):2087-97. https://doi.org/10.1038/s41593-019-0539-4 pmid: 31768052.

53. Overmyer KA, Shishkova E, Miller IJ, Balnis J, Bernstein MN, Peters-Clarke TM, et al. Large-Scale Multi-omic Analysis of COVID-19 Severity. Cell Syst. 2021; 12(1):23-40. https://doi.org/10.1016/j.cels.2020.10.003 e7. pmid: 33096026.

54. Lee JS, Park S, Jeong HW, Ahn JY, Choi SJ, Lee H, et al. Immunophenotyping of COVID-19 and influenza highlights the role of type I interferons in development of severe COVID-19. Sci Immunol. 2020;5(49). https://doi.org/10.1126/sciimmunol.abd1554 pmid: 32651212.

55. Heming M, Li X, Rauber S, Mausberg AK, Borsch AL, Hartlehnert M, et al. Neurological Manifestations of COVID-19 Feature T Cell Exhaustion and Dedifferentiated Monocytes in Cerebrospinal Fluid. Immunity. 2021;54(1): 164-75. https://doi.org/10.1016/j.immuni.2020.12.011 e6. pmid: 33382973.

56. Cheng F, Kovacs IA, Barabasi AL. Network-based prediction of drug combinations. Nat Commun. 2019;10(1):1197. https://doi.org/10.1038/s414 67-019-09186-x pmid: 30867426.

57. Smith IN, Thacker S, Seyfi M, Cheng F, Eng C. Conformational Dynamics and Allosteric Regulation Landscapes of Germline PTEN Mutations Associated with Autism Compared to Those Associated with Cancer. Am J Hum Genet. 2019;104(5):861-78. https://doi.org/10.1016/j.ajhg.2019.03.009 pmid: 31006514.

58. Huttlin EL, Ting L, Bruckner RJ, Gebreab F, Gygi MP, Szpyt J, et al. The BioPlex Network: A Systematic Exploration of the Human Interactome. Cell. 2015;162(2):425-40. https://doi.org/10.1016/j.cell.2015.06.043 pmid: 26186194.

59. Rolland T, Tasan M, Charloteaux B, Pevzner SJ, Zhong Q, Sahni N, et al. A proteome-scale map of the human interactome network. Cell. 2014;159(5): 1212-26. https://doi.org/10.1016/j.cell.2014.10.050 pmid: 25416956.

60. Rual JF, Venkatesan K, Hao T, Hirozane-Kishikawa T, Dricot A, Li N, et al. Towards a proteome-scale map of the human protein-protein interaction network. Nature. 2005;437(7062):1173-8. https://doi.org/10.1038/nature042 09 pmid: 16189514.

61. Csabai L, Olbei M, Budd A, Korcsmaros T, Fazekas D. SignaLink: Multilayered Regulatory Networks. Methods Mol Biol (Clifton, NJ). 2018;1819:53-73. https://doi.org/10.1007/978-1-4939-8618-7_3 pmid: 30421399.

62. Cheng F, Jia P, Wang Q, Zhao Z. Quantitative network mapping of the human kinome interactome reveals new clues for rational kinase inhibitor discovery and individualized cancer therapy. Oncotarget. 2014;5(11):3697710. https://doi.org/10.18632/oncotarget.1984 pmid: 25003367.

63. Keshava Prasad TS, Goel R, Kandasamy K, Keerthikumar S, Kumar S, Mathivanan S, et al. Human Protein Reference Database--2009 update. Nucleic Acids Res. 2009:37(Database issue):D767-72. https://doi.org/10.1093/ nar/gkn892 pmid: 18988627.
64. Hu J, Rho HS, Newman RH, Zhang J, Zhu H, Qian J. PhosphoNetworks: a database for human phosphorylation networks. Bioinformatics. 2014;30(1): 141-2. https://doi.org/10.1093/bioinformatics/btt627 pmid: 24227675.

65. Hornbeck PV, Zhang B, Murray B, Kornhauser JM, Latham V, Skrzypek E. PhosphoSitePlus, 2014: mutations, PTMs and recalibrations. Nucleic Acids Res. 2015;43(Database issue):D512-20. https://doi.org/10.1093/nar/gku1267 pmid: 25514926

66. Lu CT, Huang KY, Su MG, Lee TY, Bretana NA, Chang WC, et al. DbPTM 3.0: an informative resource for investigating substrate site specificity and functional association of protein post-translational modifications. Nucleic Acids Res. 2013;41(Database issue):D295-305. https://doi.org/10.1093/nar/ gks1229 pmid: 23193290.

67. Dinkel H, Chica C, Via A, Gould CM, Jensen LJ, Gibson TJ, et al. Phospho. ELM: a database of phosphorylation sites--update 2011. Nucleic Acids Res. 2011;39(Database issue):D261-7. https://doi.org/10.1093/nar/gkq1104 pmid: 21062810.

68. Oughtred R, Stark C, Breitkreutz BJ, Rust J, Boucher L, Chang C, et al. The BioGRID interaction database: 2019 update. Nucleic Acids Res. 2019;47(D1): D529-D41. https://doi.org/10.1093/nar/gky1079 pmid: 30476227.

69. Cowley MJ, Pinese M, Kassahn KS, Waddell N, Pearson JV, Grimmond SM, et al. PINA v2.0: mining interactome modules. Nucleic Acids Res. 2012; 40(Database issue):D862-5. https://doi.org/10.1093/nar/gkr967 pmid: 22067443.

70. Meyer MJ, Das J, Wang X, Yu H. INstruct: a database of high-quality 3D structurally resolved protein interactome networks. Bioinformatics. 2013; 29(12):1577-9. https://doi.org/10.1093/bioinformatics/btt181 pmid: 23599502.

71. Licata L, Briganti L, Peluso D, Perfetto L, lannuccelli M, Galeota E, et al. MINT, the molecular interaction database: 2012 update. Nucleic Acids Res. 2012; 40(Database issue):D857-61. https://doi.org/10.1093/nar/gkr930 pmid: 22096227.

72. Orchard S, Ammari M, Aranda B, Breuza L, Briganti L, Broackes-Carter F, et al. The MIntAct project--IntAct as a common curation platform for 11 molecular interaction databases. Nucleic Acids Res. 2014;42(Database issue): D358-63. https://doi.org/10.1093/nar/gkt1115 pmid: 24234451.

73. Breuer K, Foroushani AK, Laird MR, Chen C, Sribnaia A, Lo R, et al. InnateDB: systems biology of innate immunity and beyond--recent updates and continuing curation. Nucleic Acids Res. 2013;41(Database issue):D1228-33. https://doi.org/10.1093/nar/gks1147 pmid: 23180781.

74. Hagberg A, Schult D, Swart P. Exploring Network Structure, Dynamics, and Function using NetworkX. Proceedings of the 7th Python in Science Conference (SciPy2008); 2008.

75. Golbeck J. Network Structure and Measures. In: Analyzing the Social Web. Boston: Morgan Kaufmann; 2013. p. 25-44.

76. Bastian M, Heymann S, Jacomy M. Gephi: An Open Source Software for Exploring and Manipulating Networks. In: International AAAI Conference on Weblogs and Social Media; 2009.

77. GTEx Consortium. The Genotype-Tissue Expression (GTEx) project. Nat Genet. 2013;45(6):580-5. https://doi.org/10.1038/ng.2653 pmid: 23715323.

78. Virtanen P, Gommers R, Oliphant TE, Haberland M, Reddy T, Cournapeau D, et al. SciPy 1.0: fundamental algorithms for scientific computing in Python. Nat Methods. 2020;17(3):261-72. https://doi.org/10.1038/s41592-019-0686-2 pmid: 32015543.

79. Shannon P, Markiel A, Ozier O, Baliga NS, Wang JT, Ramage D, et al. Cytoscape: a software environment for integrated models of biomolecular interaction networks. Genome Res. 2003;13(11):2498-504. https://doi.org/1 0.1101/gr.1239303 pmid: 14597658.

80. Brundin P, Nath A, Beckham JD. Is COVID-19 a Perfect Storm for Parkinson's Disease? Trends Neurosci. 2020;43(12):931-3. https://doi.org/10.1016/j.tins.2 020.10.009 pmid: 33158605.

81. Zubair AS, McAlpine LS, Gardin T, Farhadian S, Kuruvilla DE, Spudich S. Neuropathogenesis and Neurologic Manifestations of the Coronaviruses in the Age of Coronavirus Disease 2019: A Review. JAMA Neurol. 2020; 77(8):1018-27. https://doi.org/10.1001/jamaneurol.2020.2065 pmid: 32469387.

82. Cook-Mills JM, Marchese ME, Abdala-Valencia H. Vascular cell adhesion molecule-1 expression and signaling during disease: regulation by reactive oxygen species and antioxidants. Antioxid Redox Signal. 2011;15(6):1607-38. https://doi.org/10.1089/ars.2010.3522 pmid: 21050132.

83. Zuliani G, Cavalieri M, Galvani M, Passaro A, Munari MR, Bosi C, et al. Markers of endothelial dysfunction in older subjects with late onset Alzheimer's 
disease or vascular dementia. J Neurol Sci. 2008;272(1-2):164-70. https://doi. org/10.1016/j.jns.2008.05.020 pmid: 18597785.

84. Huang CW, Tsai MH, Chen NC, Chen WH, Lu YT, Lui CC, et al. Clinical significance of circulating vascular cell adhesion molecule-1 to white matter disintegrity in Alzheimer's dementia. Thromb Haemost. 2015;114(6):1230-40. https://doi.org/10.1160/TH14-11-0938 pmid: 26289958.

85. Yousef H, Czupalla CJ, Lee D, Chen MB, Burke AN, Zera KA, et al. Aged blood impairs hippocampal neural precursor activity and activates microglia via brain endothelial cell VCAM1. Nat Med. 2019;25(6):988-1000. https://doi. org/10.1038/s41591-019-0440-4 pmid: 31086348.

86. Tong $\mathrm{M}$, Jiang $\mathrm{Y}$, Xia D, Xiong $\mathrm{Y}$, Zheng $\mathrm{Q}$, Chen F, et al. Elevated Expression of Serum Endothelial Cell Adhesion Molecules in COVID-19 Patients. J Infect Dis. 2020;222(6):894-8. https:/doi.org/10.1093/infdis/jiaa349 pmid: 32582936.

87. Shen J, Hou Y, Zhou Y, Mehra R, Jehi L, Cheng F. The epidemiological and mechanistic understanding of the neurological manifestations of COVID-19: a comprehensive meta-analysis and a network medicine observation. Front Neurosci. 2021;15:606926. https://doi.org/10.3389/fnins.2021.606926.

88. ten Dijke P, Hill CS. New insights into TGF-beta-Smad signalling. Trends Biochem Sci. 2004;29(5):265-73. https://doi.org/10.1016/j.tibs.2004.03.008 pmid: 15130563.

89. Li MO, Wan YY, Sanjabi S, Robertson AK, Flavell RA. Transforming growth factor-beta regulation of immune responses. Annu Rev Immunol. 2006;24(1): 99-146. https://doi.org/10.1146/annurev.immunol.24.021605.090737. pmid: 16551245.

90. Wyss-Coray T, Lin C, Yan F, Yu GQ, Rohde M, McConlogue L, et al. TGFbeta1 promotes microglial amyloid-beta clearance and reduces plaque burden in transgenic mice. Nat Med. 2001;7(5):612-8. https://doi.org/10.103 8/87945 pmid: 11329064

91. Chen JH, Ke KF, Lu JH, Qiu YH, Peng YP. Protection of TGF-beta1 against neuroinflammation and neurodegeneration in Abeta1-42-induced Alzheimer's disease model rats. PLoS One. 2015;10(2):e0116549. https://doi. org/10.1371/journal.pone.0116549 pmid: 25658940.

92. Cermakian N, Lamont EW, Boudreau P, Boivin DB. Circadian clock gene expression in brain regions of Alzheimer 's disease patients and control subjects. J Biol Rhythms. 2011;26(2):160-70. https://doi.org/10.1177/074873 0410395732 pmid: 21454296.

93. Remsik J, Wilcox JA, Babady NE, McMillen TA, Vachha BA, Halpern NA, et al. Inflammatory Leptomeningeal Cytokines Mediate COVID-19 Neurologic Symptoms in Cancer Patients. Cancer Cell. 2021;39(2):276-83. https://doi. org/10.1016/j.ccell.2021.01.007 e3. pmid: 33508216.

94. Li H, Sun X, LeSage G, Zhang Y, Liang Z, Chen J, et al. beta-arrestin 2 regulates Toll-like receptor 4-mediated apoptotic signalling through glycogen synthase kinase-3beta. Immunology. 2010;130(4):556-63. https:// doi.org/10.1111/j.1365-2567.2010.03256.x pmid: 20497256.

95. Choudhury A, Mukherjee S. In silico studies on the comparative characterization of the interactions of SARS-CoV-2 spike glycoprotein with ACE-2 receptor homologs and human TLRs. J Med Virol. 2020;92(10):210513. https://doi.org/10.1002/jmv.25987 pmid: 32383269.

96. Radzikowska U, Ding M, Tan G, Zhakparov D, Peng Y, Wawrzyniak P, et al. Distribution of ACE2, CD147, CD26, and other SARS-CoV-2 associated molecules in tissues and immune cells in health and in asthma, COPD, obesity, hypertension, and COVID-19 risk factors. Allergy. 2020;75(11):282945. https://doi.org/10.1111/all.14429 pmid: 32496587.

97. Oganesyan G, Saha SK, Guo B, He JQ, Shahangian A, Zarnegar B, et al. Critical role of TRAF3 in the Toll-like receptor-dependent and -independent antiviral response. Nature. 2006;439(7073):208-11. https://doi.org/10.1038/na ture04374 pmid: 16306936.

98. Sigrist CJ, Bridge A, Le Mercier P. A potential role for integrins in host cell entry by SARS-CoV-2. Antiviral Res. 2020;177:104759. https://doi.org/10.1016/ j.antiviral.2020.104759 pmid: 32130973.

99. Meerschaert J, Furie MB. The adhesion molecules used by monocytes for migration across endothelium include CD11a/CD18, CD11b/CD18, and VLA4 on monocytes and ICAM-1, VCAM-1, and other ligands on endothelium. J Immunol. 1995;154(8):4099-112 pmid: 7535821.

100. Daly JL, Simonetti B, Klein K, Chen KE, Williamson MK, Anton-Plagaro C, et al. Neuropilin-1 is a host factor for SARS-CoV-2 infection. Science. 2020; 370(6518):861-5. https://doi.org/10.1126/science.abd3072 pmid: 33082294.

101. Cantuti-Castelvetri L, Ojha R, Pedro LD, Djannatian M, Franz J, Kuivanen S, et al. Neuropilin-1 facilitates SARS-CoV-2 cell entry and infectivity. Science. 2020;370(6518):856-60. https://doi.org/10.1126/science.abd2985 pmid: 33082293.
102. Wang K, Chen W, Zhang Z, Deng Y, Lian JQ, Du P, et al. CD147-spike protein is a novel route for SARS-CoV-2 infection to host cells. Signal Transduction Targeted Ther. 2020;5(1):283. https://doi.org/10.1038/s41392-02 0-00426-x pmid: 33277466.

103. Pfaender S, Mar KB, Michailidis E, Kratzel A, Boys IN, V'Kovski P, et al. LY6E impairs coronavirus fusion and confers immune control of viral disease. Nat Microbiol. 2020;5(11):1330-9. https://doi.org/10.1038/s41564-020-0769-y pmid: 32704094

104. Hadjadj J, Yatim N, Barnabei L, Corneau A, Boussier J, Smith N, et al. Impaired type I interferon activity and inflammatory responses in severe COVID-19 patients. Science. 2020;369(6504):718-24. https://doi.org/10.1126/ science.abc6027 pmid: 32661059

105. Hachim MY, Al Heialy S, Hachim IY, Halwani R, Senok AC, Maghazachi AA, et al. Interferon-Induced Transmembrane Protein (IFITM3) Is Upregulated Explicitly in SARS-CoV-2 Infected Lung Epithelial Cells. Front Immunol. 2020; 11:1372. https://doi.org/10.3389/fimmu.2020.01372 pmid: 32595654.

106. Zhao X, Zheng S, Chen D, Zheng M, Li X, Li G, et al. LY6E Restricts Entry of Human Coronaviruses, Including Currently Pandemic SARS-CoV-2. J Virol. 2020;94(18). https://doi.org/10.1128/JVI.00562-20 pmid: 32641482.

107. Hur JY, Frost GR, Wu X, Crump C, Pan SJ, Wong E, et al. The innate immunity protein IFITM3 modulates gamma-secretase in Alzheimer's disease. Nature. 2020;586(7831):735-40. https://doi.org/10.1038/s41586-020-2 681-2 pmid: 32879487

108. Tay MZ, Poh CM, Renia L, MacAry PA, Ng LFP. The trinity of COVID-19: immunity, inflammation and intervention. Nat Rev Immunol. 2020;20(6):36374. https://doi.org/10.1038/s41577-020-0311-8 pmid: 32346093.

109. Qin C, Zhou L, Hu Z, Zhang S, Yang S, Tao Y, et al. Dysregulation of Immune Response in Patients With Coronavirus 2019 (COVID-19) in Wuhan. China. Clin Infect Dis. 2020;71(15):762-8. https://doi.org/10.1093/cid/ciaa248 pmid: 32161940

110. Stebbing J, Sanchez Nievas G, Falcone M, Youhanna S, Richardson P, Ottaviani S, et al. JAK inhibition reduces SARS-CoV-2 liver infectivity and modulates inflammatory responses to reduce morbidity and mortality. Sci Adv. 2021;7(1). https://doi.org/10.1126/sciadv.abe4724 pmid: 33187978.

111. Seif F, Aazami H, Khoshmirsafa M, Kamali M, Mohsenzadegan M, Pornour M, et al. JAK Inhibition as a New Treatment Strategy for Patients with COVID19. Int Arch Allergy Immunol. 2020;181(6):467-75. https://doi.org/10.1159/ 000508247 pmid: 32392562

112. Wang C, Zhang M, Garcia G Jr, Tian E, Cui Q, Chen X, et al. ApoE-IsoformDependent SARS-CoV-2 Neurotropism and Cellular Response. Cell Stem Cell. 2021;28(2):331-42. https://doi.org/10.1016/.jstem.2020.12.018 e5. pmid: 33450186.

113. Mahley RW, Rall SC Jr. Apolipoprotein E: far more than a lipid transport protein. Annu Rev Genomics Hum Genet. 2000;1(1):507-37. https://doi.org/1 0.1146/annurev.genom.1.1.507. pmid: 11701639.

114. Liu CC, Kanekiyo T, Xu H, Bu G. Apolipoprotein E and Alzheimer disease: risk, mechanisms and therapy. Nat Rev Neurol. 2013;9(2):106-18. https://doi. org/10.1038/nrneurol.2012.263 pmid: 23296339.

115. Mao XY, Jin WL. The COVID-19 Pandemic: Consideration for Brain Infection. Neuroscience. 2020;437:130-1. https://doi.org/10.1016/j.neuroscience.2020. 04.044 pmid: 32380269.

116. Gandhi S, Srivastava AK, Ray U, Tripathi PP. Is the Collapse of the Respiratory Center in the Brain Responsible for Respiratory Breakdown in COVID-19 Patients? ACS Chem Neurosci. 2020;11(10):1379-81. https://doi.org/10.1 021/acschemneuro.0c00217 pmid: 32348111.

117. Ziegler CGK, Allon SJ, Nyquist SK, Mbano IM, Miao VN, Tzouanas CN, et al. SARS-CoV-2 Receptor ACE2 Is an Interferon-Stimulated Gene in Human Airway Epithelial Cells and Is Detected in Specific Cell Subsets across Tissues. Cell. 2020;181(5):1016-35. https://doi.org/10.1016/j.cell.2020.04.035 e19. pmid: 32413319.

118. Sungnak W, Huang N, Becavin C, Berg M, Queen R, Litvinukova M, et al. SARS-CoV-2 entry factors are highly expressed in nasal epithelial cells together with innate immune genes. Nat Med. 2020;26(5):681-7. https://doi. org/10.1038/541591-020-0868-6 pmid: 32327758.

119. Lukassen S, Chua RL, Trefzer T, Kahn NC, Schneider MA, Muley T, et al. SARSCOV-2 receptor ACE2 and TMPRSS2 are primarily expressed in bronchial transient secretory cells. EMBO J. 2020;39(10):e105114. https://doi.org/10.152 52/embj.20105114 pmid: 32246845

120. Zhang H, Kang Z, Gong H, Xu D, Wang J, Li Z, et al. Digestive system is a potential route of COVID-19: an analysis of single-cell coexpression pattern of key proteins in viral entry process. Gut. 2020;69(6):1010-8. https://doi. org/10.1136/gutjnl-2020-320953. 
121. Pleasure SJ, Green AJ, Josephson SA. The Spectrum of Neurologic Disease in the Severe Acute Respiratory Syndrome Coronavirus 2 Pandemic Infection: Neurologists Move to the Frontlines. JAMA Neurol. 2020;77(6):679-80. https://doi.org/10.1001/jamaneurol.2020.1065 pmid: 32275291.

122. Arbour N, Day R, Newcombe J, Talbot PJ. Neuroinvasion by human respiratory coronaviruses. J Virol. 2000;74(19):8913-21. https://doi.org/10.112 8/jvi.74.19.8913-8921.2000 pmid: 10982334.

123. Cardinali DP, Brusco LI, Liberczuk C, Furio AM. The use of melatonin in Alzheimer's disease. Neuro Endocrinol Lett. 2002;23(Suppl 1):20-3 pmid: 12019347.

124. Cardinali DP, Furio AM, Brusco LI. Clinical aspects of melatonin intervention in Alzheimer's disease progression. Curr Neuropharmacol. 2010;8(3):218-27. https://doi.org/10.2174/157015910792246209 pmid: 21358972

125. Menche J, Sharma A, Kitsak M, Ghiassian SD, Vidal M, Loscalzo J, et al. Disease networks. Uncovering disease-disease relationships through the incomplete interactome. Science. 2015;347(6224):1257601. https://doi.org/1 $0.1126 /$ science. 1257601 pmid: 25700523.

126. Liu Y, Beyer A, Aebersold R. On the Dependency of Cellular Protein Levels on mRNA Abundance. Cell. 2016;165(3):535-50. https://doi.org/10.1016/j. cell.2016.03.014 pmid: 27104977.

\section{Publisher's Note}

Springer Nature remains neutral with regard to jurisdictional claims in published maps and institutional affiliations.

Ready to submit your research? Choose BMC and benefit from:

- fast, convenient online submission

- thorough peer review by experienced researchers in your field

- rapid publication on acceptance

- support for research data, including large and complex data types

- gold Open Access which fosters wider collaboration and increased citations

- maximum visibility for your research: over $100 \mathrm{M}$ website views per year

At BMC, research is always in progress.

Learn more biomedcentral.com/submissions 